\title{
ebv-circRPMS1 Promotes the Progression of EBV- Associated Gastric Carcinoma via Sam68 Complex Dependent Activation of METTL3
}

\section{Jing-yue Zhang}

Third Affiliated Hospital of Sun Yat-Sen University

\section{Yu Du}

Third Affiliated Hospital of Sun Yat-Sen University

\section{Li-ping Gong}

Third Affiliated Hospital of Sun Yat-Sen University

\section{Yi-ting Shao}

Sun Yat-Sen University Guanghua School of Stomatology

\section{Li-jie Pan}

Third Affiliated Hospital of Sun Yat-Sen University

\section{Zhi-ying Feng}

Third Affiliated Hospital of Sun Yat-Sen University

\section{Yu-hang Pan}

Third Affiliated Hospital of Sun Yat-Sen University

Jun-ting Huang

First Affiliated Hospital of Guangzhou Medical University

Jing-yun Wen

Third Affiliated Hospital of Sun Yat-Sen University

\section{Li-ping Sun}

Third Affiliated Hospital of Sun Yat-Sen University

\section{Gao-feng Chen}

Third Affiliated Hospital of Sun Yat-Sen University

\section{Jian-ning Chen}

Third Affiliated Hospital of Sun Yat-Sen University

Chun-Kui Shao ( $\nabla$ shaochk@mail.sysu.edu.cn)

the Third Affiliated Hospital, Sun Yat-sen University https://orcid.org/0000-0002-7083-7522

\section{Research}

Keywords: ebv-circRPMS1, Sam68, METTL3, EBV-associated gastric carcinoma, m6A modification 
Posted Date: August 31st, 2021

DOl: https://doi.org/10.21203/rs.3.rs-842156/v1

License: (c) (i) This work is licensed under a Creative Commons Attribution 4.0 International License. Read Full License

Version of Record: A version of this preprint was published at Cancer Letters on March 1st, 2022. See the published version at https://doi.org/10.1016/j.canlet.2022.215646. 


\section{Abstract}

Background: Emerging studies have showed that circular RNAs (circRNAs) are important regulators for tumorigenesis by modulating malignant behaviors of tumor cells. However, the functions of EBV-encoded circRNAs in EBV-associated gastric carcinoma (EBVaGC) remain poorly understood.

Methods: The expression of ebv-circRPMS1 in EBVaGC tissues, xenografts and cell lines were analyzed by BaseScope, qRT-PCR and in situ hybridization (ISH). The effects of ebv-circRPMS1 on gastric carcinoma (GC) cell proliferation, apoptosis, migration and invasion were measured by CCK8, EdU, immunofluorescence (IF), FACS and Transwell assays. qRT-PCR, Western blotting, ChIP, RNA fluorescence in situ hybridization (RNA-FISH), luciferase reporter assays, mass spectrum, RNA immunoprecipitation (RIP), and pulldown assays were used to investigate the molecular mechanisms. Xenograft mouse model was also used to analyze the effect of ebv-circRPMS1 on GC growth and metastasis in vivo.

Results: We demonstrated that ebv-circRPMS1 promoted the proliferation, migration, invasion and antiapoptosis of EBVaGC cells. Mechanistically, ebv-circRPMS1 recruited the Sam68 complex to the promoter of METTL3 and enhanced its transcription. Moreover, overexpression of METTL3 induced transcriptional activation of downstream genes (such as SNAI1, ZMYM1 and SOCS2) via $\mathrm{m}^{6} \mathrm{~A}$ modifications on their mRNAs, which were associated with tumor progression. Besides, RNA binding proteins (RBPs) such as QKI, DHX9 and ILF3, might involve in ebv-circRPMS1 biogenesis. In clinical EBVaGC samples, ebvcircRPMS1 was associated with distant metastasis and poor prognosis.

Conclusion: These findings indicated that ebv-circRPMS1 contributed to EBVaGC progression through recruiting the Sam68 complex to activate METTL3 expression and its downstream targets. EbvcircRPMS1, Sam68 and METTL3 may serve as therapeutic targets for EBVaGC.

\section{Background}

Epstein-Barr virus (EBV) is the first tumour virus identified in humans. It is primarily associated with a variety of lymphomas such as Hodgkin lymphoma, Burkitt lymphoma (BL) and NK/T cell lymphoma, as well as some epithelial cancers such as nasopharyngeal carcinoma (NPC) and gastric carcinoma (GC) ${ }^{1}$. EBV-encoded small non-coding RNAs (EBERs, including EBER1 and EBER2) could be detected by in situ hybridization (ISH) in about $10 \%$ of GC worldwide, which are defined as EBV-associated GC (EBVaGC) ${ }^{2,3}$. In addition to EBERs, EBV expresses microRNAs (ebv-miR-BARTs), the BamHI A region long non-coding RNAs, EBV nuclear antigen 1 (EBNA1) and/or latent membrane 2A (LMP2A) in EBVaGC. Recently, it has been shown that EBV also produces circular RNAs (ebv-circRNAs) ${ }^{4,5}$. For example, ebv-circRPMS1, which is derived from the RPMS1 gene, was found to be expressed in B-cell lymphoma, NPC and EBVaGC ${ }^{6,7}$. A lower abundance of the circEBNA_U, which derived from the EBNA locus, was detected in B cells that displayed type I or type III latency or underwent reactivation ${ }^{8}$. While recent studies have revealed that EBV generates a diverse repertoire of viral circRNAs, the functions of these viral circRNAs remain largely unknown. Our group demonstrated that ebv-circLMP2A was enriched in cancer stem cells (CSCs) of 
EBVaGC and involved in inducing and maintaining stemness phenotypes through targeting the miR3908/TRIM59/p53 axis ${ }^{9}$. Liu et al. showed that ebv-circRPMS1 promoted epithelial-mesenchymal transition (EMT) in NPC through sponging multiple miRNAs, such as miR-203, miR-31 and miR-4517.

$\mathrm{N}^{6}$-methylation of adenosine $\left(\mathrm{m}^{6} \mathrm{~A}\right)$ is the most abundant modification ubiquitously occurred in eukaryotic mRNAs, which plays an important role in the regulation of mRNA stability, splicing, transport, localization and translation ${ }^{10,11}$. The $\mathrm{m}^{6} \mathrm{~A}$ modification is dynamic and reversible in mammalian cells. It can be installed by $\mathrm{m}^{6} \mathrm{~A}$ methyltransferases, such as METTL3, METTL14 and WTAP, and removed by $\mathrm{m}^{6} \mathrm{~A}$ demethylases including FTO and ALKBH5. Additionally, specific RNA-binding proteins (RBPs), such as YTHDF1/2/3, YTHDC1/2, elF3, IGF2BP1/2/3 and HNRNPA2B1, can bind to the $\mathrm{m}^{6} \mathrm{~A}$ motifs directly or indirectly to affect RNA function ${ }^{12,13}$. It has been reported that $\mathrm{m}^{6} \mathrm{~A}$ modification is involved in various biological processes, including stem cell differentiation, tissue development, and tumor progression ${ }^{14-17}$.

To date, little is known about the roles that ebv-circRNAs play in the development of EBVaGC. In this study, we found that ebv-circRPMS1, an EBV-encoded circRNA derived from back-splicing of RPMS1 (from exon 4 to exon 2), was highly expressed in EBVaGC tissues and positively correlated with distant metastasis and poor outcome. In addition, ebv-circRPMS1 promoted EBVaGC cell proliferation, migration and invasion, and inhibited apoptosis in vitro and in vivo. Mechanistically, ebv-circRPMS1 recruited the Sam68 complex to the promoter of METTL3, resulting in METTL3 transcription enhancement and subsequent activation of downstream genes (such as SNAI1, ZMYM1 and SOCS2) via $\mathrm{m}^{6} \mathrm{~A}$ modification on their mRNAs. These findings indicated that ebv-circRPMS1 contributed to EBVaGC progression through Sam68 complex dependent activation of METTL3, a key enzyme of $\mathrm{m}^{6} \mathrm{~A}$ modification.

\section{Materials And Methods}

\section{Cell lines}

EBV-positive gastric cell line SNU719 was purchased from Korean Cell Line Bank. EBV-positive gastric cell line YCCEL1 was provided by Prof. Qian Tao from The Chinese University of Hong Kong, Hong Kong, China. EBV-negative gastric cell lines BGC823 and AGS were purchased from Cell Bank of Type Culture Collection of Chinese Academy of Sciences (Shanghai, China). EBV-positive lymphoma cell line Akata was a gift from Prof. Mu-sheng Zeng from Cancer Center of Sun Yat-sen University. HEK-293T cells were provided by Dr. Jun Zheng from the Third Affiliated Hospital of Sun Yat-sen University. AGS-EBV was generated by AGS cells co-cultured with EBV-positive Akata cells, as previously described by Yue et al ${ }^{18}$. All cells except HEK-293T cells were cultured in RPMI-1640 medium (Gibco, Carlsbad, CA, USA) supplemented with 10\% FBS (Gibco), while HEK-293T cells were maintained in Dulbecco's Modified Eagle's Medium (Gibco) supplemented with 10\% FBS. All cells were grown in a humidified incubator at 37 ${ }^{\circ} \mathrm{C}$ with $5 \% \mathrm{CO}_{2}$.

\section{RNA interference (RNAi), plasmid construction and transfection}


siRNAs for knockdown of ebv-circRPMS1, METTL3, Sam68, DHX9, ILF3 and QKI were designed by Hanbio (Shanghai, China). The sequences of siRNAs are listed in Supplementary Table S1. ebvcircRPMS1 overexpression plasmid was obtained from GENESEED (Guangzhou, China). METTL3, Sam68 and P53 overexpression plasmids were obtained from RiboBio (Guangzhou, China). Transfections of siRNAs or plasmids into GC cells were performed with Lipofectamine 3000 kit (Invitrogen) according to the manufacturer's instructions. For stable transfections, lentiviral vectors were first co-transfected with packaging plasmids psPAX2, pMD2G and CD513B-1 into HEK-293T cells. Infectious recombinant lentiviruses were harvested at 48 and 72 hours after transfection and filtered through $0.45 \mu \mathrm{m}$ filters, then concentrated 100-fold by ultracentrifugation (2 hours at 120,000 x g). Finally, the recombinant lentiviruses were transfected into GC cells according to the manufacturer's instructions. Puromycin was used for two weeks to select stable cells.

\section{Quantitative real-time PCR (qRT-PCR)}

Total RNA was extracted using TRIzol reagent (Invitrogen). Reverse transcription was performed using the Transcriptor First Strand cDNA Synthesis Kit (Takara). Quantitative real-time PCR (qRT-PCR) was carried out using SYBR Green SuperMix (Takara) in ABI7500 Fast Real-Time PCR system (Applied Biosystems). The relative expression of mRNAs was calculated using the $2^{-\triangle \Delta C t}$ method. GAPDH or U6 was used as the endogenous control to normalize the data. All primer sequences are shown in Supplementary Table S2 and Table S3.

\section{Actinomycin D and RNase R treatment}

Cells were planted into six-well plates, up to $60 \%$ confluency after $24 \mathrm{~h}$, cells were treated with Actinomycin D ( $5 \mu \mathrm{g} / \mathrm{ml}$, Millipore) or DMSO and collected at $0 \mathrm{~h}, 4 \mathrm{~h}, 8 \mathrm{~h}, 12 \mathrm{~h}$ and $24 \mathrm{~h}$. Total RNA $(2 \mu \mathrm{g})$ was incubated with $3 \mathrm{U} / \mu \mathrm{g}$ of RNase R (Epicentre Technologies) for 20 min at $37^{\circ} \mathrm{C}$. After treatment with Actinomycin D or RNase R, the levels of ebv-circRPMS1 and RPMS1 mRNAs were analyzed by qRT-PCR.

\section{Nuclear and cytoplasmic extraction}

Cytoplasmic and nuclear fractions were isolated using the reagents supplied in PARIS ${ }^{\text {TM }}$ Kit (Thermo Fisher Scientific). Briefly, SNU719 cells were lysed in Cell Fraction Buffer on ice for 10 min. After centrifugation at $500 \times \mathrm{g}$ for $3 \mathrm{~min}$ at $4{ }^{\circ} \mathrm{C}$, the supernatant was collected as cytoplasmic fraction. Then, by washing the pellet with Cell Fraction Buffer, the nuclei fraction was collected.

\section{Western blotting}

Proteins were lysed with $1 \times$ cell lysis buffer (KeyGEN BioTECH, Nanjing, China). Equal amounts of protein were separated by SDS-PAGE at $80 \mathrm{~V}$ for $2.5 \mathrm{~h}$ and transferred to PVDF membranes for $2 \mathrm{~h}$. After incubation with antibodies (1:1000 dilution) at $4{ }^{\circ} \mathrm{C}$ overnight, the membranes were hybridized with a secondary antibody at RT for $1 \mathrm{~h}$. The immunoreactive signals were visualized by enhanced chemiluminescence kit (Millipore). All antibodies are shown in Supplementary Table S4. 


\section{Protein stability of METTL3}

Cells were treated with cycloheximide (CHX, $100 \mu \mathrm{g} / \mathrm{ml}, 0,2$ and $4 \mathrm{~h})$ or MG132 $(20 \mu \mathrm{M}, 0,4$ and $8 \mathrm{~h})$. The expression of METTL3 was measured by western blotting.

\section{Cell proliferation assays}

Cell proliferation was evaluated by 5-Ethynyl-20-deoxyuridine (EdU) incorporation assay and/or Cell Counting Kit-8 (CCK8) assay. The EdU assay was performed using a Cell-Light EdU DNA Cell Proliferation Kit (RiboBio, Shanghai, PR, China) according to the manufacturer's instructions. For CCK8 assay, $1 \times 10^{4}$ cells were seeded in 96-well plates, cells were then treated with CCK-8 reagent (10 $\mu \mathrm{L} /$ well, Dojindo, Japan) at $24 \mathrm{~h}, 36 \mathrm{~h}, 72 \mathrm{~h}, 96 \mathrm{~h}$ and $120 \mathrm{~h}$, and incubated for $2 \mathrm{~h}$ at $37^{\circ} \mathrm{C}$. Absorbance was measured at 450 nm using a microplate reader.

\section{Migration assay}

Migration assays were carried out using 24-well Corning Transwell Chambers (Corning) according to the manufacturer's recommendations. Briefly, $2 \times 10^{4}$ cells in $200 \mu \mathrm{l}$ of serum-free medium were added to the inner chambers, and 1640 medium containing $20 \%$ FBS was added in the bottom chamber. After incubation for $24 \mathrm{~h}$, the migrated cells in lower filters were fixed in methanol, stained with $0.1 \%$ crystal violet (Sigma, MO) and counted under microscope.

\section{Matrigel invasion assay}

Invasion assays were carried out using 24-well Corning Transwell Chambers (Corning) according to the manufacturer's recommendations. Briefly, $5 \times 10^{4}$ cells in $200 \mu \mathrm{l} 1640$ medium were added to the inner chambers of Matrigel-coated wells. Medium containing $20 \%$ FBS was added to the bottom chamber. The cells were incubated for $36 \mathrm{~h}$ at $37^{\circ} \mathrm{C}$. Cells on the lower surface of the membrane were stained with $0.1 \%$ crystal violet (Sigma) and counted under microscope.

\section{Flow cytometry analysis of cell apoptosis}

Cells were planted into six-well plates, up to $80 \%$ confluency, cells were treated with 5 -Fluorouracil at the concentration of $2.5 \mu \mathrm{M}$ or $5 \mu \mathrm{M}$. At 48 hours after culture, cells were stained with Annexin V-PE and 7-AAD (BD) according to the manufacturer's instructions. The cells were analyzed by using a BD FacScanto II flow cytometer and analyzed with FlowJo software.

\section{RNA fluorescence in situ hybridization (RNA-FISH)}

RNA-FISH was performed following the manufacturer's instructions. Firstly, the probes targeting the backsplicing site of ebv-circRPMS1 were designed by RiboBio (Guangzhou, China). Cells were fixed with $4 \%$ paraformaldehyde for $10 \mathrm{~min}$ and then permeabilized in PBS with $0.5 \%$ Triton X-100 for $5 \mathrm{~min}$. Next, the cells were hybridized with the labeled ebv-circRPMS1 probes at $37^{\circ} \mathrm{C}$ overnight. Afterwards, the cells were 
washed with $4 \times$ sodium citrate buffer containing $0.1 \%$ Tween- 20 for 5 min and then $1 \times$ SSC for 5 min. Finally, cells were stained with DAPI for $10 \mathrm{~min}$. The images were acquired using a laser confocal microscope (Leica).

\section{Fluorescence immunocytochemical staining}

Cells were seeded on glass coverslips in 24-well plates. Cells were fixed with $4 \%$ paraformaldehyde at room temperate for $30 \mathrm{~min}$ and incubated with antibodies specific for SAM68 or P53. Then, coverslips were treated with Alexa Fluor 594 or 488 goat anti-rabbit lgG, whereas nuclei were counterstained using DAPI. Images were acquired using a laser confocal microscope (Leica).

\section{RNA tag-labeled assay}

The RNA-protein complex was pulled down by incubating the cell lysates with RNA tag-labeled-coupled MS2 (GENESEED) according to a previous study ${ }^{19}$. The level of ebv-circRPMS1 in the complex was evaluated by qRT-PCR, whereas the proteins in the complex were detected by western blotting or mass spectrometry.

\section{RNA-binding protein immunoprecipitation (RIP)}

RIP assay was performed using a Magna RIP RNA-Binding Protein Immunoprecipitation Kit (Millipore) according to the manufacturer's instructions. Briefly, cells were lysed in RIP lysis buffer on ice for $30 \mathrm{~min}$. After centrifugation, the supernatant was incubated with $30 \mu$ of Protein-A/G magnetic beads (MedChemExpress) and antibodies. After incubation overnight, the immune complexes were centrifuged then washed six times with $1 \times$ washing buffer. The beads bound proteins were further analyzed using western blotting. The immunoprecipitated RNA was subjected to qRT-PCR analysis.

\section{Co-immunoprecipitation (Co-IP)}

Cells were incubated in lysis buffer containing protease inhibitor Cocktail (MedChemExpress) for subsequent Co-IP. The cell lysates were incubated with specific antibodies targeting Flag, METTL3, SAM68, MS2 and P53 at $4{ }^{\circ} \mathrm{C}$ for $2 \mathrm{~h}$, then incubated with $30 \mu$ Protein-A/G magnetic beads at $4{ }^{\circ} \mathrm{C}$ overnight. The beads were separated and washed by using cold phosphate-buffered saline, and then subjected to western blotting analysis.

\section{ChIP assays}

Briefly, cells were crosslinked with formaldehyde for $15 \mathrm{~min}$ and terminated with $0.125 \mathrm{M}$ glycine. Cells were then sonicated to generate chromatin with average size of $500 \mathrm{bp}$. Samples were first pre-cleared with Protein-G magnetic beads (Cell Signaling) for $1 \mathrm{~h}$ at $4{ }^{\circ} \mathrm{C}$. Cleared chromatin was then incubated with the antibodies overnight at $4{ }^{\circ} \mathrm{C}$, and then with Protein-A/G magnetic beads for $6 \mathrm{~h}$ at $4{ }^{\circ} \mathrm{C}$. Beads were then recovered, washed multiple times, and de-cross-linked by incubating them overnight at $65^{\circ} \mathrm{C}$ in $1 \%$ SDS, $0.1 \mathrm{M} \mathrm{NaHCO}_{3}$. DNA was then purified by using the E.Z.N.A. Gel Extraction Kit DNA (Omega). The 
promoters of the specific genes were then analyzed by qRT-PCR. Primer sequences used for qRT-PCR are included in Supplementary Table S2.

\section{Luciferase reporter assay}

The sequence of METTL3 promoter was cloned downstream of p-GLO Dual-Luciferase vector (VectorBuilder). 293T cells in 96 well plates were transfected with luciferase reporter and indicated expression constructs. All cells were harvested $48 \mathrm{~h}$ after transfection and analyzed using the dualluciferase reporter gene assay system (Promega, Madison, WI). The relative ratio of firefly luciferase activity to Renilla luciferase activity was determined.

\section{$\mathrm{m}^{6} \mathrm{~A}$ RNA immunoprecipitation (MeRIP) assay}

$\mathrm{m}^{6} \mathrm{~A}$ RNA immunoprecipitation (MeRIP) assay was conducted according to the previously described protocol with a slight modification ${ }^{20}$. Briefly, $100 \mathrm{ng}$ of the total RNA was collected as input; the remaining RNA was used for $\mathrm{m}^{6} \mathrm{~A}$-immunoprecipitation with $\mathrm{m}^{6} \mathrm{~A}$ antibody and Protein $\mathrm{A} / \mathrm{G}$ to obtain $\mathrm{m}^{6} \mathrm{~A}$ pull down portion. Finally, immunoprecipitated RNA was analyzed through qRT-PCR.

\section{Total $\mathrm{m}^{6} \mathrm{~A}$ RNA quantification}

The EpiQuik $\mathrm{m}^{6} \mathrm{~A}$ RNA Methylation Quantification Kit (Colorimetric) was used to measure the $\mathrm{m}^{6} \mathrm{~A}$ level of total RNA in GC cells. First, 200ng RNA was added into the assay wells. Then, the detection antibody solution was added into assay wells. The $\mathrm{m}^{6} \mathrm{~A}$ level was quantified by colorimetry, and the absorbance of each well was measured at $450 \mathrm{~nm}$, and the calculation of $\mathrm{m}^{6} \mathrm{~A}$ level according to the standard curve.

\section{$\mathrm{m}^{6} \mathrm{~A}$ dot blot assay}

Firstly, a serial dilution of RNA was spotted onto a HybondN+ membrane (GE Healthcare). The membranes were then UV crosslinked, blocked, incubated with $\mathrm{m}^{6} \mathrm{~A}$ antibody and horseradish peroxidase conjugate anti-rabbit immunoglobulin G. Finally, the signals from the dot blot were visualized by an ECL Western Blotting Detection Kit (Thermo Fisher Scientific). Methylene blue staining served as a loading control.

\section{Animal studies}

NOD/SCID mice were obtained from GemPharmatech Laboratory (Nanjing, China). For the xenograft model, $3 \times 10^{6}$ cells were injected into the subcutaneous of mouse ( $n=5$ per group). For the in vivo lung metastases model, $5 \times 10^{6}$ cells suspended in $100 \mu$ PBS were injected intravenously into the tail vein of each 4-week-old NOD/SCID mice ( $n=5$ per group). After eight weeks, mice were anaesthetized with isoflurane and images were acquired with the Xenogen IVIS Lumina series II for 5 min and analyzed using the Living Image 2.11 software package (Xenogen Corp). All the mice were sacrificed after eight weeks, and the xenografts and lungs were fixed with phosphate-buffered formalin and sectioned for H\&E 
staining and immunohistochemical analysis. All animal studies were performed in accordance with the institutional ethics guidelines for the animal experiments which were approved by the Experimental Animal Ethics Committee of the Third Affiliated Hospital, Sun Yat-sen University.

\section{Patient specimens}

Human EBVaGC tissues were collected from 70 patients who received surgery at The Third Affiliated Hospital of Sun Yat-sen University from 2000 to 2012. Patients' clinical data and pathological features were obtained from medical records. The tumor grade and stage were defined according to the World Health Organization (WHO) classification and the eighth edition of TNM classification of the International Union Against Cancer (UICC). All the patients were followed up on a regular basis, and the overall survival (OS) time was determined from the date of surgery to the date of death or the date of the last follow-up visit for survivors. Informed consents were obtained from all participants before surgery. All specimens were obtained with appropriate informed consent from the patients and approved by the Institute Research Ethics Committee of Sun Yat-Sen University.

\section{Immunohistochemistry and in situ hybridization}

Immunohistochemistry (IHC) analysis was performed using a GT Vision III Kit (Leica) according to the manufacturer's instructions. The staining results were scored as follows: staining intensity score, 0 (no staining), 1 (weak), 2 (moderate), or 3 (strong); staining area score, 0 ( $\leq 10 \%$ positive staining), 1 (11-50\% positive staining), 2 (51-75\% positive staining), and 3 ( $\geq 75 \%$ positive staining). Staining intensity score and staining area score were multiplied to yield a final score. Finally, the expression was divided into low and high expression group according to ROC curve. In situ hybridization (ISH) assay was performed with a commercially available EBV oligonucleotide probe complementary to EBER-1 (PanPath, Amsterdam, Netherlands), as previously described by Chen et $\mathrm{al}^{21}$.

\section{BaseScope assay}

BaseScope assays were performed in accordance with the guidelines provided by the manufacturer (Advanced Cell Diagnostics). $4 \mu \mathrm{m}$ thick sections were placed onto Superfrost plus slides (Fisher Scientific) and baked at $60^{\circ} \mathrm{C}$ for $1 \mathrm{~h}$ before deparaffinizing in xylene $(2 \times 5 \mathrm{~min})$ and ethanol $(2 \times 2 \mathrm{~min})$. After drying by baking at $60^{\circ} \mathrm{C}$ for $2 \mathrm{~min}$, pretreat 1 (hydrogen peroxide) was applied for $10 \mathrm{~min}$ at RT, followed by Pretreat 2 (target retrieval) for $30 \mathrm{~min}$ at $100^{\circ} \mathrm{C}$ and Pretreat 3 (protease) for $30 \mathrm{~min}$ (tissue sections) or $15 \mathrm{~min}$ (cell pellets) at $40^{\circ} \mathrm{C}$, with two rinses in distilled water between pretreatments. EbvcircRPMS1 probes were then incubated for $2 \mathrm{~h}$ at $40^{\circ} \mathrm{C}$ in a HybEZ oven before incubation with reagents AMP0 (30 min at $\left.40^{\circ} \mathrm{C}\right), \operatorname{AMP} 1\left(15 \mathrm{~min}\right.$ at $\left.40^{\circ} \mathrm{C}\right), \mathrm{AMP} 2\left(30 \mathrm{~min}\right.$ at $\left.40^{\circ} \mathrm{C}\right), \mathrm{AMP} 3\left(30 \mathrm{~min}\right.$ at $\left.40^{\circ} \mathrm{C}\right), \mathrm{AMP} 4$ $\left(15 \mathrm{~min}\right.$ at $\left.40^{\circ} \mathrm{C}\right)$, AMP5 (30 min at RT) and AMP6 (15 min at RT). Slides were rinsed with wash buffer (2 $\times 2 \mathrm{~min}$ ) between each AMP incubation. Finally, slides were incubated with Fast Red for $10 \mathrm{~min}$ at RT in the dark. Slides were counterstained with Gill's hematoxylin and mounting in VectaMount permanent mounting medium. 


\section{Statistical analysis}

All experiments were carried out at least three times. Data are presented as mean \pm standard deviation (S.D.). The statistical significance of differences was evaluated by Chi-square test, two-tailed Student's ttest or one-way ANOVA. OS was assessed with the Kaplan-Meier method and compared by the Log-rank test. The correlations among ebv-circRPMS1, METTL3 and SAM68 expression in xenografts and EBVaGC tissues were calculated by Pearson correlation analysis. Statistical significance was set at a value of $\mathrm{P}<$ 0.05. All statistical analyses were carried out using SPSS 22.0.

\section{Results}

\section{1. ebv-circRPMS1 promotes EBVaGC cell proliferation, migration, invasion, and inhibits apoptosis}

Ebv-circRPMS1 is a circRNA that encoded by EBV ${ }^{6,8,22}$. To further characterize ebv-circRPMS1, we designed divergent primers to detect the circRNA through RT-PCR and Sanger sequencing (Fig.S1B). The results revealed that ebv-circRPMS1 was derived from back splicing between exon 4 and exon 2 of RPMS1 (Fig. S1A). RNase R treatment, which degrades linear transcripts but not circular ones, showed that the linear transcript (RPMS1 mRNA) was dramatically reduced, while ebv-circRPMS1 remained untouched (Fig. S1C). In addition, the half-life of ebv-circRPMS1 exceeded $24 \mathrm{~h}$, whereas that of RPMS1 mRNA was about $8 \mathrm{~h}$ (Fig. S1D), indicating that ebv-circRPMS1 is more stable than its cognate mRNA. Moreover, ebv-circRPMS1 localized both in the cytosol and the nucleus (Fig. S1E-F). Taken together, these data indicated that ebv-circRPMS1 is a bona fide circRNA.

In order to investigate the potential roles of ebv-circRPMS1 in EBVaGC tumorigenesis, ebv-circRPMS1 expressing vector was constructed by cloning the circularizing sequence into the vector, which favored the formation of ebv-circRPMS1 (Fig. 1A). The nuclear localization of ebv-circRPMS1 was further analyzed by RNA fluorescent in-situ hybridization assay in ebv-circRPMS1-transfected AGS cells. RNA FISH clearly demonstrated ebv-circRPMS1 exclusively in the nucleus (Fig. S2). Ectopic expression of ebvcircRPMS1 promoted cell proliferation, migration and invasion, and inhibited apoptosis in AGS and BGC823 cells (Fig. 1B-H, Fig. S3 \& Fig. S4). In contrast, effectively knocked down the expression of endogenous ebv-circRPMS1 by small interfering RNAs (siRNAs) targeting the ebv-circRPMS1 backsplice junction (Fig. S5A), significantly inhibited the proliferation, migration and invasion of SNU719 and YCCEL1 cells, and induced more apoptosis (Fig. S5B-F). These results demonstrated that ebv-circRPMS1 might participate in EBVaGC tumorigenesis through promoting cell migration and invasion.

\section{2. ebv-circRPMS1 regulates EBVaGC cell malignant behaviors through activating METTL3}

Given that $\mathrm{m}^{6} \mathrm{~A}$ methylation is potentially involved in tumor progression ${ }^{17}$, we examined the level of total $\mathrm{m}^{6} \mathrm{~A}$ methylation in AGS and BGC823 cells with ebv-circRPMS1 overexpression. Compared with mock controls, the RNA $\mathrm{m}^{6} \mathrm{~A}$ levels were significantly higher in ebv-circRPMS1 overexpression cells (Fig. 2A-B). Next, we examined the expressions of $\mathrm{m}^{6} \mathrm{~A}$ related enzymes by qRT-PCR and western blot. METTL3 was 
the only enzyme that was up-regulated in GC cells with ebv-circRPMS1 overexpression (Fig. 2C-D, Fig. S6).

Overexpression of METTL3 significantly upregulate the $\mathrm{m}^{6} \mathrm{~A}$ levels on RNA in AGS and BGC823 cells (Fig. $3 A-D)$. In addition, it enhanced the proliferation, migration and invasion of AGS and BGC823 cells, and inhibited apoptosis (Fig. 3E-I, Fig. S7 \& Fig. S8). On the contrary, stable METTL3 knockdown in SNU719 and YCCEL1 cells dramatically reduced the $\mathrm{m}^{6} \mathrm{~A}$ levels on RNA (Fig. S9 \& Fig. S10A-D). Besides, knockdown of METTL3 obviously suppressed GC cell proliferation, migration and invasion and induces apoptosis in SNU719 and YCCEL1 cells (Fig.S10E-I).

Taken together, these findings suggested that ebv-circRPMS1 regulates EBVaGC cell malignant behaviors through METTL3.

\section{3. ebv-circRPMS1 directly interacts with Sam68 complex to activate METTL3 transcription}

The most acknowledged function of circRNAs is a microRNA sponge function, while we failed to identify miRNA interactions with ebv-circRPMS1 (Fig. S11), it suggests that the viral circRNA probably do not function as miRNA sponges. To further investigate the molecular mechanisms, RNA tag-labeled pulldown was performed using MS2-labeled protein (Fig. 4A, Fig. S12). Mass spectrometry revealed that 48 proteins consistently pulled down by exogenous ebv-circRPMS1 (Fig. 4B), and 8 of them were RBPs defined by RBPDB. Further comprehensive analysis indicated Sam68 was the potential ebv-circRPMS1interacting partners (Fig. 4C-E, Fig. S13). The RNA recognition motif domain [174-202 amino acids (aa)] but not amino- or carboxyl-terminus of Sam68 protein was necessary for its interaction with ebvcircRPMS1 (Fig. 4F). Mutation of RNA recognition motif domain, potential interacting regions analyzed by catRAPID, abolished the interaction of Sam68 with ebv-circRPMS1 (Fig. 4G). In addition, Sam68 was significantly up-regulated with ebv-circRPMS1 overexpression (Fig. 4H). These results suggested that ebvcircRPMS1 directly interacted with Sam68 protein in EBV positive cells.

Furthermore, the present study investigated how ebv-circRPMS1 targets and inhibits the expression of METTL3 gene. By treating with cycloheximide (CHX) and MG132, it was revealed that ebv-circRPMS1 did not affect the protein stability of METTL3(Fig. S14), indicating that ebv-circRPMS1 may affect the protein level of METTL3 at post-transcriptional level. Sam68 is a transcriptional regulator that activates downstream gene expression ${ }^{23}$. Therefore, we wondered whether Sam68 participates in the regulation of METTL3 transcription. ChIP assay showed that Sam68 enriched on METTL3 promoter region (-800 -600 bp from the transcription start site) (Fig. 5A, Fig. S15). Notably, ebv-circRPMS1 silencing impaired the enrichment of Sam68 on METTL3 promoter (Fig. 5A). Additionally, ebv-circRPMS1 silencing suppressed the enrichment of transcriptional active marker H3K27ac on METTL3 promoter (Fig. 5B). Moreover, P53, a cofactor reported to interact with Sam68, which can synergistically activate the transcription and regulate gene expression ${ }^{23}$, enriched on METTL3 promoter region (Fig. 5C-D). Also, ebvcircRPMS1 colocalized on METTL3 promoter with Sam68 (Fig. 5E). Overexpression of ebv-circRPMS1, Sam68 and P53, respectively, promoted the luciferase activity of METTL3 promoter (Fig. 5F), 
demonstrating that ebv-circRPMS1, Sam68 and P53 may form a complex to promote METTL3 transcription.

In ebv-circRPMS1 overexpression AGS cells, silencing of Sam68 decreased the expression of METTL3 and the RNA $\mathrm{m}^{6} \mathrm{~A}$ levels, and reversed the effects of ebv-circRPMS1 overexpression on cell proliferation, apoptosis, migration and invasion. In ebv-circRPMS1-depleted YCCEL1 cells, upregulation of Sam68 significantly increased the expression of METTL3 and the RNA ${ }^{6}{ }^{6}$ levels, and reversed the effects of ebv-circRPMS1 silencing on cell proliferation, apoptosis, migration and invasion (Fig. 6, Fig. S16 \& Fig. S17). These data suggested that ebv-circRPMS1 directly interacted with Sam68 complex to activate METTL3 transcription, thus promoting tumor progression.

\section{SNAl1, ZMYM1 and SOCS2 are potential genes involved in EBVaGC progression}

Since METTL3 is an $\mathrm{m}^{6} \mathrm{~A}$ writer, we sought to find out its potential targets involved in $\mathrm{m}^{6} \mathrm{~A}$-regulated tumor progression. According to the previous studies ${ }^{15,24,25}$, eight genes (SNAI1, ZMYM1, SOCS2, HDGF, MALAT1, MET, ADAT2, TGFB1) were selected as candidate genes. The $\mathrm{m}^{6} \mathrm{~A}$ levels of Snail (encoded by SNAI1), ZMYM1 and SOCS2 mRNAs were significantly increased in ebv-circRPMS1 expression cells than in negative controls (Fig. S18). In addition, their mRNA levels were also higher in ebv-circRPMS1 overexpression cells than those in ebv-circRPMS1 knockdown cells (Fig. S18).

\section{Effects of ebv-circRPMS1 on EBVaGC growth in vivo}

In NOD/SCID mice, ebv-circRPMS1 overexpression significantly increased tumor volumes and weights, while ebv-circRPMS1 knockdown showed the opposite results (Fig. 7A). The protein levels of METTL3, Ki67 and Sam68 were found to be significantly higher in ebv-circRPMS1 overexpression xenografts than in mock xenografts (Fig. 7B-D), while the expression of P53 in xenografts of each group unchanged (Fig. S19). Besides, the expressions of ebv-circRPMS1 and Ki-67, METTL3, Sam68 were positively correlated in xenografts (Fig. S20). RNA-FISH assay showed the colocalization between ebv-circRPMS1 and Sam68 in xenograft tumor tissues (Fig. S21). Furthermore, stable overexpression of ebv-circRPMS1 group displayed more lung metastatic tumors than ebv-circRPMS1 knockdown group (Fig. 7E).

\section{Up-regulated ebv-circRPMS1 in EBVaGC tissues positively correlated with poor prognosis}

In EBVaGC, the expression of ebv-circRPMS1 was positively correlated with the expression of METTL3 (Fig. 8A-B) and distant metastasis ( $p=0.001$, Table 1). Besides, the expressions of METTL3 and Sam68 were also positively correlated (Fig. 8B). In human gastric carcinoma samples, RNA-FISH showed that ebv-circRPMS1 and Sam68 co-localize in the nucleus (Fig. S22). The expression of METTL3 was significantly correlated with lymph node metastasis $(p=0.023)$ and distant metastasis $(p=0.006)$, while the expression of Sam68 was significantly correlated with Lauren classification $(p=0.030)$, lymph node metastasis $(p=0.013)$ and distant metastasis $(p=0.017)$ (Table 1). Furthermore, higher expression of ebvcircRPMS1 in EBVaGC patients predicted shorter overall survival (Fig. 8C). The patients with METTL3 up- 
regulation also had a worse overall survival (Fig. $8 \mathrm{C}$ ). These results indicated that ebv-circRPMS1 exerts an oncogenic role in EBVaGC.

\section{Identification of potential splicing regulators in ebv-circRPMS1 biogenesis}

QKI, DHX9 and ILF3 (NF90/NF110) are RBPs that have been identified as splicing-associated factors involved in circRNA biogenesis ${ }^{26-28}$. Knockdown of these three RBPs in YCCEL1 cell line showed a reduction of ebv-circRPMS1; however, the expression of RPMS1 mRNA was not affected (Fig. 9A). Therefore, QKI, DHX9, and ILF3 might involve in ebv-circRPMS1 biogenesis.

\section{Discussion}

In this study, we characterized the function and molecular mechanisms of ebv-circRPMS1 in EBVaGC. We found that ebv-circRPMS1 promoted EBVaGC cell proliferation, migration and invasion and inhibited apoptosis both in vitro and in vivo. Mechanistically, ebv-circRPMS1 recruited the Sam68 complex to METTL3 promoter, which subsequently activated the transcription of METTL3 and regulated downstream genes that associated with tumor progression, such as SNAIL, ZMYM1 and SOCS2, via $\mathrm{m}^{6} \mathrm{~A}$ modifications on their mRNAs. In addition, we demonstrated that QKI, DHX9 and ILF3 might involve in ebv-circRPMS1 biogenesis. In clinical EBVaGC samples, ebv-circRPMS1 was highly expressed in EBVaGC tissues and predicted poor prognosis. These findings indicated that ebv-circRPMS1 contributed to EBVaGC progression through recruiting the Sam68 complex to activate METTL3 expression and its downstream targets.

Recent studies have found that EBV, a human DNA tumor virus, produced circRNAs $6,8,9,22$. ebv-circRPMS1 was found to be expressed in a panel of EBV-associated diseases representing latency types I, II, and III, including EBV-positive PTLD, BL, EBVaGC, NPC, and AIDS-associated lymphoma ${ }^{6,8,22}$. In addition, ebvcircRPMS1 was also expressed during reactivation ${ }^{29}$. ebv-circEBNA_U, which derived from the EBNA locus, was detected in B cells that displayed type I or type III latency or underwent reactivation ${ }^{29}$. ebvcircBHLF1 was detected in most latency cell models but displayed extraordinarily high expression under reactivation conditions ${ }^{29}$. In contrast, ebv-circLMP2 was seldom expressed in latency, but expressed specifically during reactivation ${ }^{22}$.

Despite extensive identification of EBV circRNAs, the function of EBV circRNAs has not been extensively explored. Our group has demonstrated that ebv-circLMP2A was enriched in cancer stem cells (CSCs) of EBVaGC and could induce and maintain stemness through targeting the miR-3908/TRIM59/p53 axis ${ }^{9}$. Here, we showed that ebv-circRPMS1 was stably expressed in EBVaGC and played an essential role in EBVaGC progression. Ectopic expression of ebv-circRPMS1 enhanced GC cell proliferation, migration and invasion and inhibited apoptosis. Similarly, Liu et al. found that ebv-circRPMS1 promoted tumor metastasis in NPC, probably through sponging multiple miRNAs, such as miR-203, miR-31 and miR-4517. In EBVaGC, we also tried to see whether ebv-circRPMS1 could also act as a miRNA sponge. Although 
some miRNA binding sites of ebv-circRPMS1 were predicted by bioinformative software, we were unable to identify interactions between ebv-circRPMS1 and these cellular miRNAs (Fig. S9).

Considering the finding that ebv-circRPMS1 was mainly located in the nucleus of EBVaGC cells, we wondered whether ebv-circRPMS1 exerted its function through RNA-protein interaction, because most nuclear circRNAs regulate gene expression at post-transcriptional or transcriptional levels through RNAprotein interaction. For example, cis-acting circ-CTNNB1 acted as a mediator of $\beta$-catenin signaling through DDX3-mediated transactivation of $Y Y 1$ and promoted cancer progression ${ }^{30}$. Circ-DONSON was localized in the nucleus, recruited the NURF complex to SOX4 promoter and initiated its transcription in gastric cancer cells ${ }^{31}$. CircSamd4 bound with PUR proteins and thereby prevented their interaction with the Mhc promoter, which repressed myogenic transcriptional activity ${ }^{32}$. In the present study, we found that ebv-circRPMS1 can bind to RNA-binding protein Sam68, and regulate METTL3 transcription.

Sam68 is a transcription factor involved in a variety of cellular processes, including alternative splicing, cell cycle regulation, RNA 3'-end formation, tumorigenesis, and regulation of human immunodeficiency virus (HIV) gene expression ${ }^{33,34}$. Sam68 usually acts as a potent transcriptional co-factor in tumor progression via interacting with transcription regulatory proteins, such as $\mathrm{P} 53^{23}$. As a transcriptional regulator, Sam68 has been reported to regulate the promoter activity of NF-KB/P65 $5^{35}$. In this study, we demonstrate that Sam68 functions as a transcription co-factor with P53 to facilitate the transactivation of METTL3. In addition, we found that RNA recognition motif domain of Sam68 was necessary for its interaction with ebv-circRPMS1. Mutation of RNA recognition motif domain abolished the interaction of Sam68 with ebv-circRPMS1.

METTL3, a methyltransferase of $m R N A ~ m^{6} A$, exhibits functions for the self-renewal of cancer stem cells, promotion of cancer cell proliferation, and resistance to radiotherapy or chemotherapy ${ }^{36,37}$. We found that METTL3 expression was dramatically up-regulated in ebv-circRPMS1 expression cells and promoted GC cell proliferation, migration and invasion in an ebv-circRPMS1-dependent manner. Studies have found that $\mathrm{m}^{6} \mathrm{~A}$ modifications were associated with latent and lytic infection of virus ${ }^{38}$. Xia et al. showed that $\mathrm{N}^{6}$-methyladenosine $\left(\mathrm{m}^{6} \mathrm{~A}\right)$-binding protein $\mathrm{YTHDF} 1$ suppresses EBV replication and promotes EBV RNA decay ${ }^{39}$. Blocking $\mathrm{m}^{6} \mathrm{~A}$ inhibited splicing of the pre-mRNA encoding replication transcription activator (RTA) and halted lytic replication of $\mathrm{KSHV}^{40}$. Depletion of the $\mathrm{m}^{6} \mathrm{~A}$ machinery had different anti-viral impacts on gene expression of KSHV depending on the cell-type analyzed ${ }^{41}$. In this study, we showed for the first time that the host RNA $\mathrm{m}^{6} \mathrm{~A}$ modification machinery can be targeted and manipulated specifically by a virus-encoded circRNA, ebv-circRPMS1. It suggested a novel link between the host epitranscriptome and EBV-mediated oncogenesis.

So far, there are three well-studied mechanisms about circRNA biogenesis ${ }^{42}$. The first one is that RBPs binding to intronic regions and facilitating their pairing through homo- or hetero-dimerization promote circRNA biogenesis. The second one is that the presence of flanking inverted Alu repeat elements that stabilize proximity of the splice donor and acceptor sequences ease circRNA formation. The last one, the

Page $14 / 31$ 
orientation-opposite complementary sequences residing in the flanking introns of the circularized exon(s) facilitated the formation of circRNA. In the present study, knockdown of QKI, ILF3, and DHX9 repressed circRNA biogenesis. QKI is a dimer, promotes circRNA biogenesis by bringing the circle-forming exons into close proximity. It is essential for enhanced production of many circRNAs and acts by binding to recognition elements within introns, in the vicinity of the circRNA-forming splice sites ${ }^{27}$. ILF3 is a dsRNA binding protein, which strongly bind to and stabilize transient RNA pairs formed between intronic complementary sequences, leading to the enhanced circRNA biogenesis in the nucleus ${ }^{26}$. DHX9 is a helicase which stabilizes internal repeat Alu pairing and promotes circRNA biogenesis ${ }^{28}$. Our data suggested that RNA binding/processing factors such as these RBPs play an important role in facilitating EBV backsplicing, however, the exact mechanisms still need to be further investigated.

In conclusion, we found that ebv-circRPMS1 promoted EBVaGC cell proliferation, migration and invasion and inhibited apoptosis. Mechanistically, ebv-circRPMS1 interacted with Sam68 to activate METTL3, an $\mathrm{m}^{6} \mathrm{~A}$ writer of mRNA, which regulated the expression of genes associated cancer progression, such as SNAIL, ZMYM1 and SOCS2, via $\mathrm{m}^{6} \mathrm{~A}$ modification of their mRNA. In addition, ebv-circRPMS1 was associated with distant metastasis and unfavorable outcome in clinical EBVaGC samples. Taken together, these findings indicated that ebv-circRPMS1 contributed to EBVaGC progression through recruiting the Sam68 complex to activate METTL3 expression and its downstream targets.

\section{Conclusion}

This study not only extends our knowledge about the regulation of $m R N A m^{6} A$ modification by virusencoded circRNA, but also implies that targeting the ebv-circRPMS1/Sam68-METTL3 axis might be a promising strategy for treatment of EBVaGC.

\section{Abbreviations}




\begin{tabular}{|ll|}
\hline Abbreviation & Full name \\
\hline EBV & Epstein-Barr virus \\
\hline EBVaGC & Epstein-Barr virus-associated gastric carcinoma \\
\hline EBVnGC & Epstein-Barr virus-negative gastric carcinoma \\
\hline EBERs & Epstein-Barr virus encoded small RNAs \\
\hline GC & Gastric carcinoma \\
\hline OS & Overall survival \\
\hline IHC & Immunohistochemistry \\
\hline RIP & RNA immunoprecipitation \\
\hline RBP & RNA binding protein \\
\hline qRT-PCR & Quantitative real-time PCR \\
\hline m ${ }^{6}$ A & N 6 -methyladenosine \\
\hline METTL3 & Methyltransferase-like 3 \\
\hline METTL14 & methyltransferase-like 14 \\
\hline FTO & Fat-mass and obesity-associated protein \\
\hline ALKBH5 & Alkylation repair homolog protein 5 \\
\hline MeRIP & m ${ }^{6}$ A-RNA Immunoprecipitation \\
\hline Co-IP & Co-immunoprecipitation \\
\hline SOCS2 & suppressor of cytokine signaling 2 \\
\hline KHDRBS1 & KH domain containing, RNA binding, signal transduction associated 1 \\
\hline CHX & cycloheximide \\
\hline SNAl1 & snail family transcriptional repressor 1 \\
\hline ZMYM1 & zinc finger MYM-type containing 1 \\
\hline MALAT1 & metastasis associated lung adenocarcinoma transcript 1 \\
\hline ADAT2 & adenosine deaminase tRNA specific 2 \\
\hline
\end{tabular}

\section{Declarations}




\section{Ethics approval and consent to participate}

The present study was approved by the Institute Research Ethics Committee of Sun Yat-Sen University.

\section{Consent for publication}

Consent was achieved from all patients.

\section{Availability of data and materials}

All data are included in the manuscript.

Conflicts of interest

The authors declare no potential conflicts of interest

\section{Funding}

This work was supported by the National Natural Science Foundation of China (82073397), the Natural Science Foundation of Guangdong Province (2019A1515011455, 2018A030313650), the Guangzhou Science and Technology Project (202102010156) and the NSFC cultivating grant of The Third Affiliated Hospital, Sun Yat-sen University (2020GZRPYMS01), Guangdong Provice, China.

\section{Author contributions}

$\mathrm{J}-\mathrm{yZ}$ designed the study, performed the experiments and wrote the manuscript. J-nC performed part of the experiments and revised the manuscript, YD and L-pG interpreted part of the data and revised the manuscript. Z-yF, Y-hP and L-jP performed IHC Scoring and provided statistics of data. Y-tS and J-yW involved in data curation and formal analysis. J-tH, L-pS and G-fC involved in collection of clinical specimens. C-kS designed the study and revised the manuscript.

\section{Acknowledgements}

We sincerely thank Prof. Qian Tao from The Chinese University of Hong Kong, Hong Kong, China for providing the EBV-positive gastric cell line YCCEL1, Prof. Mu-sheng Zeng from Cancer Center of Sun Yatsen University for providing the EBV-positive lymphoma cell line Akata, and Dr. Jun Zheng from the Third Affiliated Hospital of Sun Yat-sen University for providing the HEK-293T cell line.

\section{Author details}

${ }^{1}$ Department of Pathology, The Third Affiliated Hospital, Sun Yat-sen University, Guangzhou 510630, China

${ }^{2}$ Guanghua School of Stomatology \& Guangdong Provincial Key Laboratory of Stomatology, Sun Yat-sen University, Guangzhou 510055, China

${ }^{3}$ Vaccine Research of Sun Yat-sen University, Guangzhou 510630, China 
${ }^{4}$ The First Affiliated Hospital of Guangzhou Medical University, Guangzhou 510182, China

${ }^{5}$ Department of Medical Oncology, The Third Affiliated Hospital, Sun Yat-sen University, Guangzhou 510630, China

\section{References}

1. Farrell, P. J. Epstein-Barr Virus and Cancer. Annu. Rev. Pathol. 14, 29-53, doi:10.1146/annurevpathmechdis-012418-013023 (2019).

2. Zhang, J. et al. 3-The oncogenic role of Epstein-Barr virus-encoded microRNAs in Epstein-Barr virusassociated gastric carcinoma. J. Cell. Mol. Med. 22, 38-45, doi:10.1111/jcmm.13354 (2018).

3. Zebardast, A., Tehrani, S. S., Latifi, T. \& Sadeghi, F. Critical review of Epstein-Barr virus microRNAs relation with EBV-associated gastric cancer. Journal of cellular physiology, doi:10.1002/jcp.30297 (2021).

4. Nahand, J. S. et al. Circular RNAs: New Epigenetic Signatures in Viral Infections. Front Microbio/11, 1853, doi:10.3389/fmicb.2020.01853 (2020).

5. Lei, F. et al. Radio-Susceptibility of Nasopharyngeal Carcinoma: Focus on Epstein-Barr virus, MicroRNAs, Long non-coding RNAs and Circular RNAs. Curr. Mol. Pharmacol., doi:10.2174/1874467213666191227104646 (2019).

6. Huang, J.-T. et al. Identification of virus-encoded circular RNA. Virology 529, 144-151, doi:10.1016/j.virol.2019.01.014 (2019).

7. Liu, Q., Shuai, M. \& Xia, Y. Knockdown of EBV-encoded circRNA circRPMS1 suppresses nasopharyngeal carcinoma cell proliferation and metastasis through sponging multiple miRNAs. Cancer Manag. Res. 11, 8023-8031, doi:10.2147/cmar.S218967 (2019).

8. Ungerleider, N. A. et al. Comparative Analysis of Gammaherpesvirus Circular RNA Repertoires: Conserved and Unique Viral Circular RNAs. J. Virol. 93, doi:10.1128/jvi.01952-18 (2019).

9. Gong, L. P. et al. Epstein-Barr virus-derived circular RNA LMP2A induces stemness in EBV-associated gastric cancer. EMBO Rep 21, e49689, doi:10.15252/embr.201949689 (2020).

10. Jiang, X. et al. The role of m6A modification in the biological functions and diseases. Signal Transduct Target Ther 6, 74, doi:10.1038/s41392-020-00450-x (2021).

11. Zhou, Z. et al. Mechanism of RNA modification N6-methyladenosine in human cancer. Mol. Cancer 19, 104, doi:10.1186/s12943-020-01216-3 (2020).

12. Huang, $\mathrm{H}$. et al. Recognition of RNA N-methyladenosine by IGF2BP proteins enhances mRNA stability and translation. Nat. Cell Biol. 20, 285-295, doi:10.1038/s41556-018-0045-z (2018).

13. Li, B. et al. circNDUFB2 inhibits non-small cell lung cancer progression via destabilizing IGF2BPs and activating anti-tumor immunity. Nature communications 12, 295, doi:10.1038/s41467-020-20527-z (2021). 
14. Chen, X.-Y., Zhang, J. \& Zhu, J.-S. The role of mA RNA methylation in human cancer. Mol. Cancer 18, 103, doi:10.1186/s12943-019-1033-z (2019).

15. Lee, H. et al. Stage-specific requirement for Mettl3-dependent m(6)A mRNA methylation during haematopoietic stem cell differentiation. Nat. Cell Biol. 21, 700-709, doi:10.1038/s41556-019-0318-1 (2019).

16. Wang, T., Kong, S., Tao, M. \& Ju, S. The potential role of RNA N6-methyladenosine in Cancer progression. Mol. Cancer 19, 88, doi:10.1186/s12943-020-01204-7 (2020).

17. He, L. et al. Functions of N6-methyladenosine and its role in cancer. Mol. Cancer 18, 176, doi:10.1186/s12943-019-1109-9 (2019).

18. Yue, W. et al. Early Pattern of Epstein-Barr Virus Infection in Gastric Epithelial Cells by "Cell-in-cell". Virol. Sin. 34, 253-261, doi:10.1007/s12250-019-00097-1 (2019).

19. A game of tag MAPS catches up on RNA interactomes. doi:10.1080/15476286.2016.1156830.

20. Yue, B. et al. METTL3-mediated N6-methyladenosine modification is critical for epithelialmesenchymal transition and metastasis of gastric cancer. Mol. Cancer 18, 142, doi:10.1186/s12943019-1065-4 (2019).

21. Chen, J.-N. et al. Association of distinctive Epstein-Barr virus variants with gastric carcinoma in Guangzhou, southern China. J. Med. Virol. 82, 658-667, doi:10.1002/jmv.21731 (2010).

22. Toptan, T. et al. Circular DNA tumor viruses make circular RNAs. Proc. Natl. Acad. Sci. U. S. A. 115, E8737-E8745, doi:10.1073/pnas.1811728115 (2018).

23. Li, N. \& Richard, S. Sam68 functions as a transcriptional coactivator of the p53 tumor suppressor. Nucleic Acids Res. 44, 8726-8741 (2016).

24. Lin, X. et al. RNA mA methylation regulates the epithelial mesenchymal transition of cancer cells and translation of Snail. Nature communications 10, 2065, doi:10.1038/s41467-019-09865-9 (2019).

25. Wang, Q. et al. METTL3-mediated m6A modification of HDGF mRNA promotes gastric cancer progression and has prognostic significance. (2019).

26. Li, X. et al. Coordinated circRNA Biogenesis and Function with NF90/NF110 in Viral Infection. Molecular cell 67, 214-227.e217, doi:10.1016/j.molcel.2017.05.023 (2017).

27. Conn, S. J. et al. The RNA binding protein quaking regulates formation of circRNAs. Cell 160, 11251134, doi:10.1016/j.cell.2015.02.014 (2015).

28. Aktaş, T. et al. DHX9 suppresses RNA processing defects originating from the Alu invasion of the human genome. Nature 544, 115-119, doi:10.1038/nature21715 (2017).

29. Ungerleider, N. et al. The Epstein Barr virus circRNAome. PLoS Pathog. 14, e1007206, doi:10.1371/journal.ppat.1007206 (2018).

30. Yang, F. et al. Acting Promotes $\beta$-Catenin Signaling and Cancer Progression via DDX3-Mediated Transactivation of YY1. 79, 557-571, doi:10.1158/0008-5472.CAN-18-1559 (2019).

31. Ding, L. et al. Circular RNA circ-DONSON facilitates gastric cancer growth and invasion via NURF complex dependent activation of transcription factor SOX4. Mol. Cancer 18, 45, doi:10.1186/s12943- 
019-1006-2 (2019).

32. Pandey, P. R. et al. circSamd4 represses myogenic transcriptional activity of PUR proteins. Nucleic Acids Res., doi:10.1093/nar/gkaa035 (2020).

33. Naro, C. et al. Functional Interaction between U1snRNP and Sam68 Insures Proper 3 ' End Pre-mRNA Processing during Germ Cell Differentiation. Cell reports 26, doi:10.1016/j.celrep.2019.02.058 (2019).

34. Sánchez-Jiménez, F. \& Sánchez-Margalet, V. Role of Sam68 in post-transcriptional gene regulation. International journal of molecular sciences 14, 23402-23419, doi:10.3390/ijms141223402 (2013).

35. Qian, J., Zhao, W., Miao, X., Li, L. \& Zhang, D. Sam68 modulates apoptosis of intestinal epithelial cells via mediating NF-kB activation in ulcerative colitis. Mol. Immunol. 75, 48-59, doi:10.1016/j.molimm.2016.05.011 (2016).

36. Chen, X.-Y., Zhang, J. \& Zhu, J.-S. 6-The role of mA RNA methylation in human cancer. Mol. Cancer 18, 103, doi:10.1186/s12943-019-1033-z (2019).

37. Yi, Y.-C., Chen, X.-Y., Zhang, J. \& Zhu, J.-S. Novel insights into the interplay between mA modification and noncoding RNAs in cancer. Mol. Cancer 19, 121, doi:10.1186/s12943-020-01233-2 (2020).

38. Wu, F. et al. Association of N6-methyladenosine with viruses and related diseases. Virol J. 16, doi:10.1186/s12985-019-1236-3 (2019).

39. Xia, T. L. et al. N(6)-methyladenosine-binding protein YTHDF1 suppresses EBV replication and promotes EBV RNA decay. EMBO Rep 22, doi:10.15252/embr.202050128 (2021).

40. Ye, F., Chen, E. R. \& Nilsen, T. W. Kaposi's Sarcoma-Associated Herpesvirus Utilizes and Manipulates RNA N-Adenosine Methylation To Promote Lytic Replication. J. Virol. 91, doi:10.1128/JVI.00466-17 (2017).

41. Zheng, X. et al. RNA m6A methylation regulates virus-host interaction and EBNA2 expression during Epstein-Barr virus infection. Immunity, Inflammation and Disease 9, 351-362, doi:10.1002/iid3.396 (2021).

42. Chen, L.-L. The expanding regulatory mechanisms and cellular functions of circular RNAs. Nature reviews. Molecular cell biology 21, 475-490, doi:10.1038/s41580-020-0243-y (2020).

\section{Tables}

Table 1. Expression of ebv-circRPMS1, Sam68 and METTL3 in relation to patients' characteristics in 70 cases of EBVaGC 


\begin{tabular}{|c|c|c|c|c|c|c|c|c|c|}
\hline \multirow[b]{2}{*}{ Characteristics } & \multicolumn{3}{|c|}{ ebv-circRPMS1 } & \multicolumn{3}{|c|}{ METTL3 } & \multicolumn{3}{|c|}{ Sam68 } \\
\hline & high & low & $P$ & high & low & $P$ & high & low & $P$ \\
\hline No. of cases & 31 & 39 & & 36 & 34 & & 42 & 28 & \\
\hline \multicolumn{10}{|l|}{ Age } \\
\hline$<60$ years & 16 & 25 & 0.945 & 26 & 15 & 0.156 & 26 & 15 & 0.051 \\
\hline$\geq 60$ years & 15 & 14 & & 10 & 19 & & 16 & 13 & \\
\hline \multicolumn{10}{|l|}{ Sex } \\
\hline Male & 28 & 37 & 0.451 & 33 & 32 & 0.806 & 38 & 27 & 0.556 \\
\hline Female & 3 & 2 & & 3 & 2 & & 4 & 1 & \\
\hline \multicolumn{10}{|l|}{ Size } \\
\hline$<5 \mathrm{~cm}$ & 12 & 19 & 0.992 & 10 & 11 & 0.235 & 20 & 12 & 0.082 \\
\hline$\geq 5 \mathrm{~cm}$ & 19 & 20 & & 26 & 13 & & 22 & 17 & \\
\hline \multicolumn{10}{|l|}{ Differentiation } \\
\hline Well/Moderate & 8 & 3 & 0.063 & 9 & 2 & 0.321 & 8 & 3 & 0.633 \\
\hline Poor & 23 & 36 & & 27 & 32 & & 34 & 25 & \\
\hline \multicolumn{10}{|c|}{ Lauren classification } \\
\hline Intestinal & 10 & 5 & 0.593 & 12 & 3 & 0.263 & 6 & 9 & 0.030 \\
\hline Diffuse & 21 & 34 & & 24 & 31 & & 36 & 19 & \\
\hline \multicolumn{10}{|l|}{ T stage } \\
\hline $\mathrm{T} 1+\mathrm{T} 2$ & 4 & 2 & 0.100 & 4 & 2 & 0.055 & 2 & 4 & 0.398 \\
\hline $\mathrm{T} 3+\mathrm{T} 4$ & 27 & 37 & & 32 & 22 & & 40 & 24 & \\
\hline \multicolumn{10}{|c|}{ Lymph node status } \\
\hline Negative & 8 & 10 & 0.247 & 6 & 12 & 0.023 & 7 & 11 & 0.013 \\
\hline Positive & 23 & 29 & & 30 & 22 & & 35 & 17 & \\
\hline \multicolumn{10}{|c|}{ Distant metastasis } \\
\hline Negative & 18 & 31 & 0.001 & 20 & 29 & 0.006 & 27 & 22 & 0.017 \\
\hline Positive & 13 & 8 & & 16 & 5 & & 15 & 6 & \\
\hline
\end{tabular}


A

ebv-circRPMS 1 construct

ebv-circRPMS
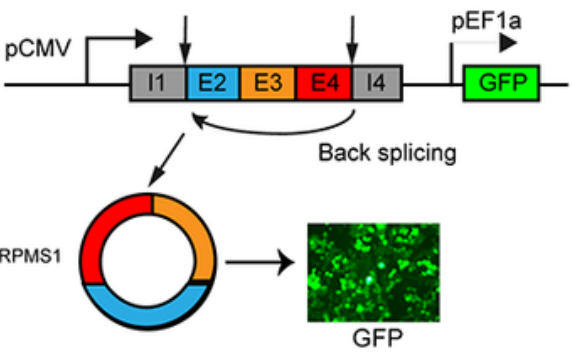

B

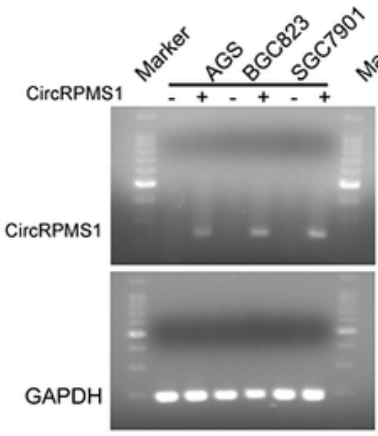

E
C

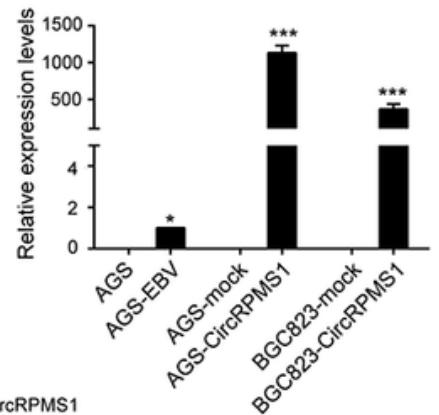

D AGS
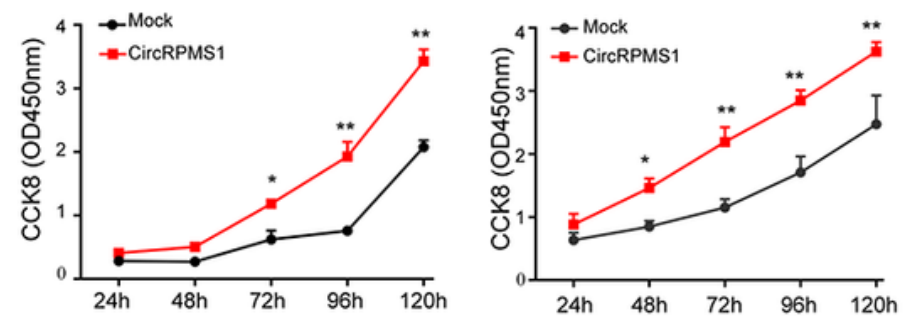

F

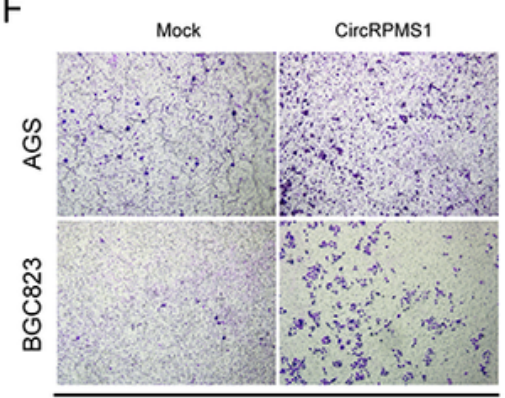

Migration

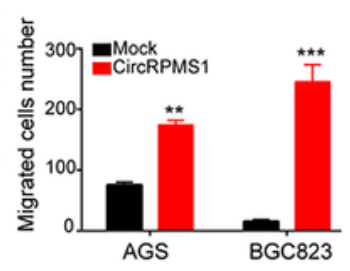

$\mathrm{H}$

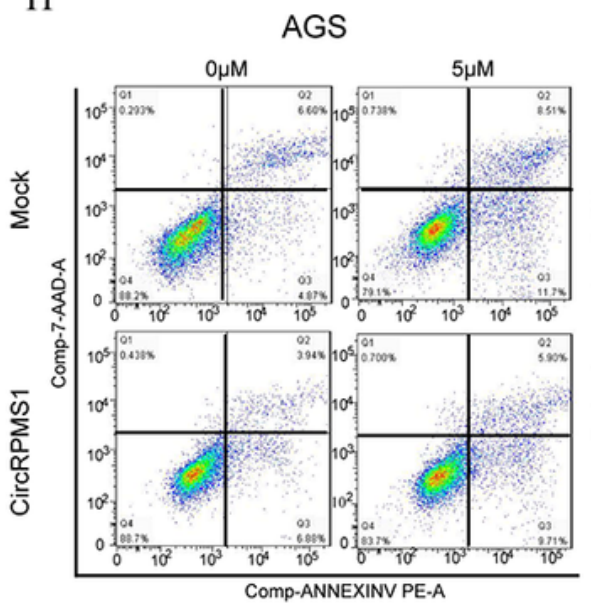

G
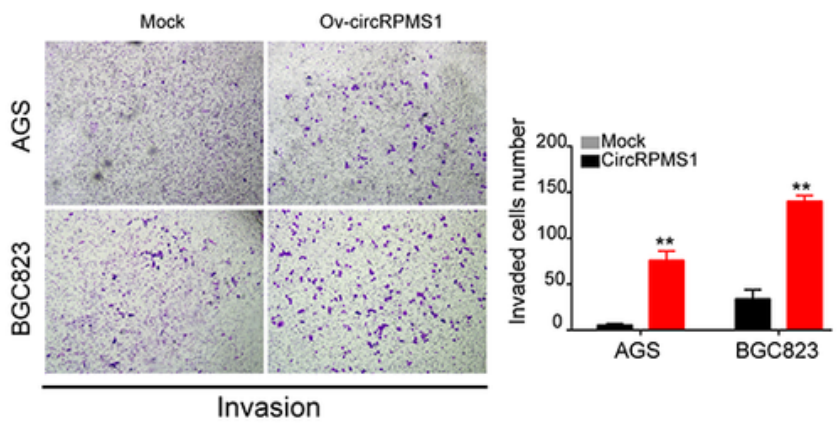

BGC823
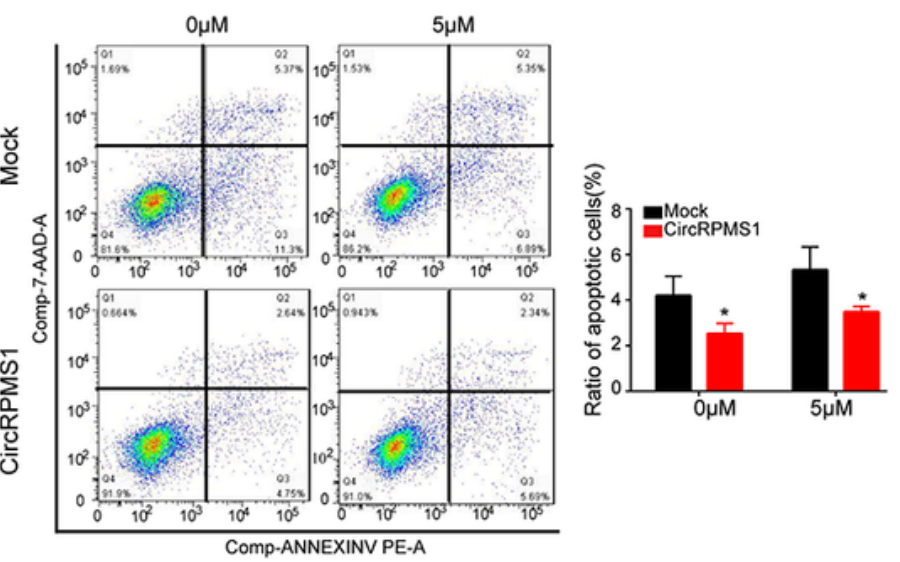

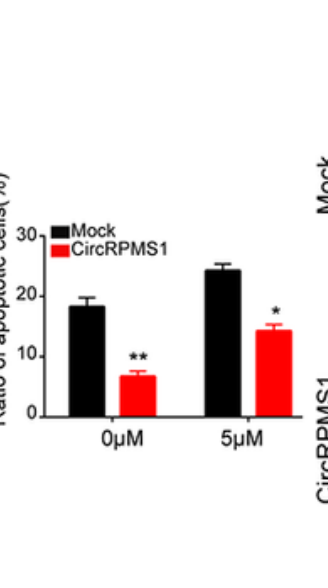

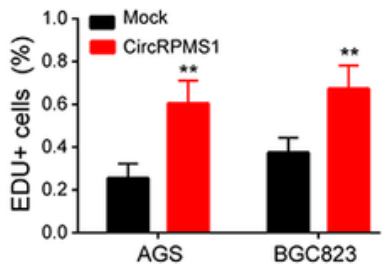

Figure 1

ebv-circRPMS1 promotes GC cell proliferation, migration and invasion, and inhibits apoptosis. A, the ebvcircRPMS1 expression cassette is composed of RPMS1 exons 4-2-3 and the intron fragments containing the back-splicing elements. pCMV, CMV promoter; pEF1a, EF1a promoter. GFP fluorescent marker was 
used to track the transfection. B and C, RT-PCR and qRT-PCR assay revealing the relative levels of ebvcircRPMS1 (normalized to GAPDH) in GC cell lines. D and E, CCK8 and EdU assays were used to analyze the proliferation of AGS and BGC823 cells after ebv-circRPMS1 overexpression. Scale bar, $200 \mu \mathrm{m}$. F and G, Transwell assay illustrated that ebv-circRPMS1 overexpression facilitated the migration and invasion of AGS and BGC823 cells. Scale bars, $20 \mu \mathrm{m}$. H, Annexin/PI staining followed by FACS analysis indicated that ebv-circRPMS1 overexpression inhibited apoptosis. ${ }^{\star} p<0.05,{ }^{* \star} p<0.01,{ }^{* \star *} p<0.001$

A

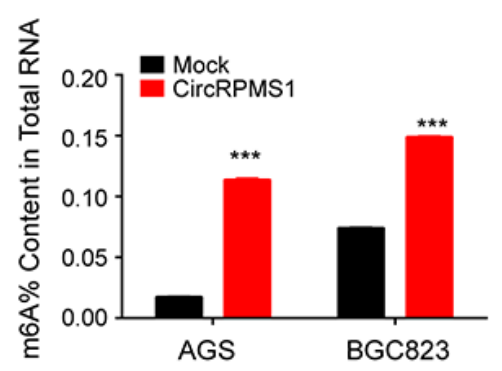

C

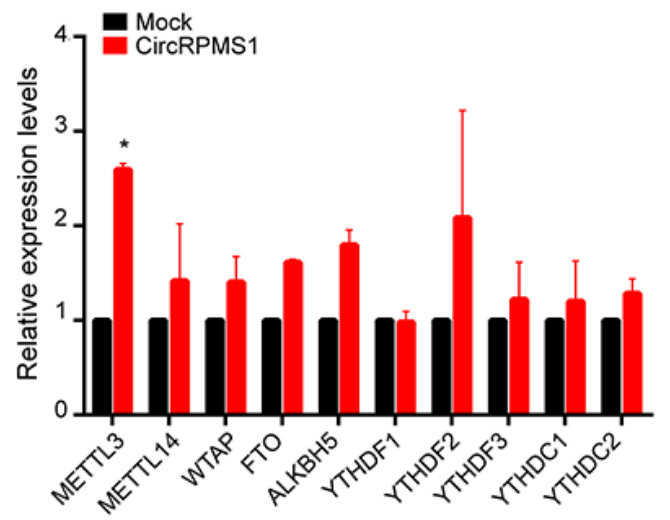

B

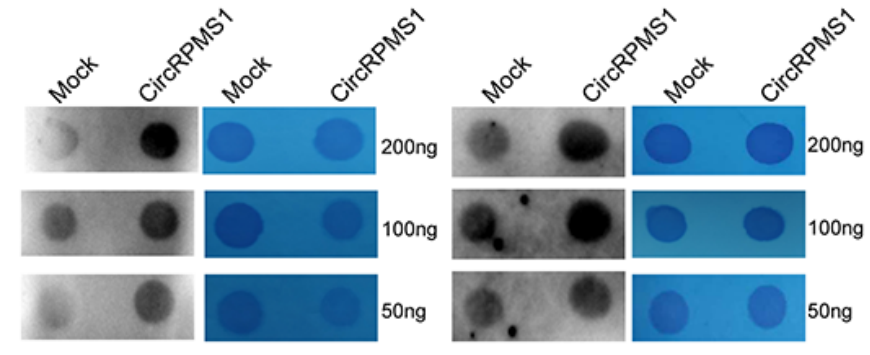

BGC823

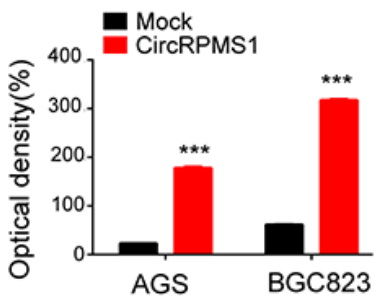

$\mathrm{D}$
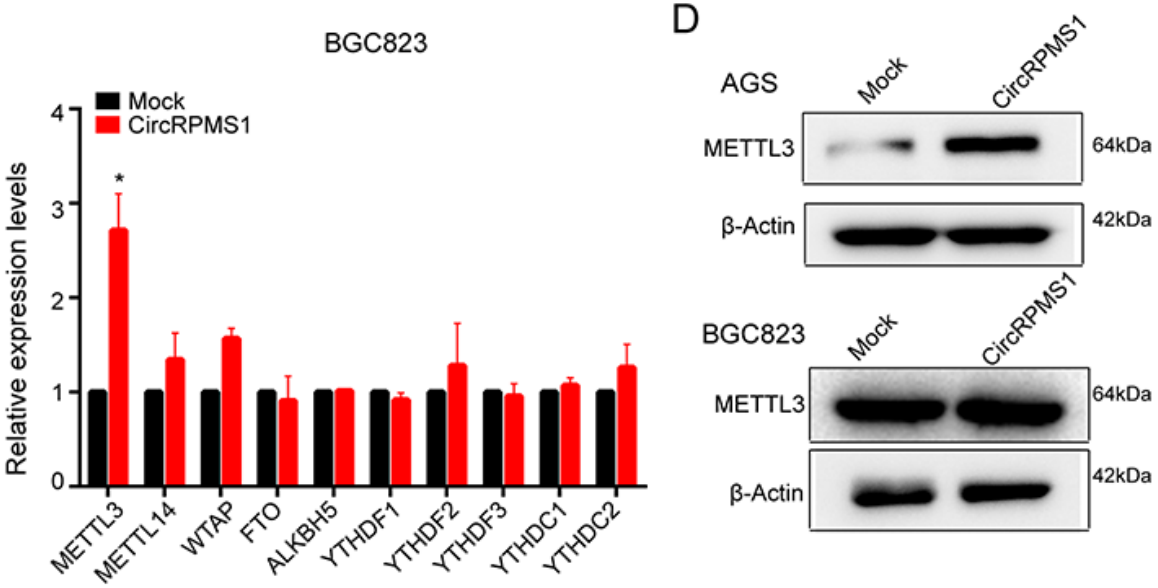

Figure 2

ebv-circRPMS1 increases the expression of m6A level on RNA and the expression of METTL3 in vitro. A, the m6A levels on RNA were detected by colorimetric ELISA-like assay using an RNA m6A methylation quantification kit. B, the m6A levels on RNA were detected by dot blot analyses with an anti-m6A antibody. Methylene blue (MB) staining served as the loading control. Relative m6A contents on total RNA were calculated. C, the expressions of m6A-related enzymes in GC cells were measured by qRT-PCR. D, METTL3 protein in GC cells was detected by western blotting. ${ }^{*} p<0.05$, ${ }^{* \star} \mathrm{p}<0.001$ 
A

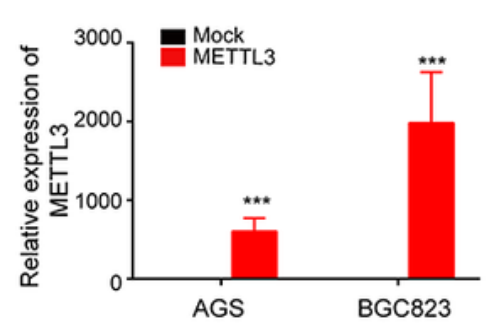

E
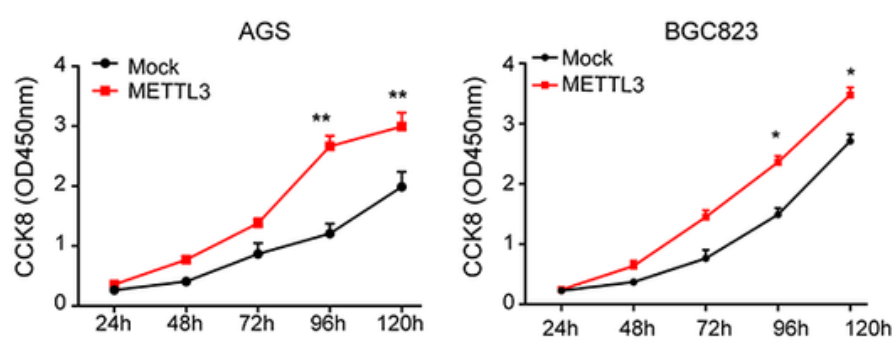

B

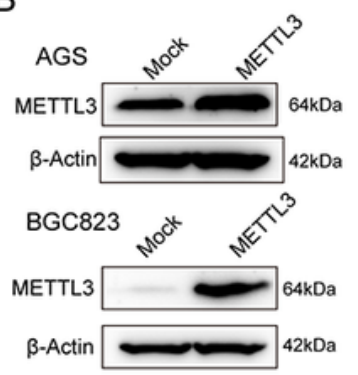

C

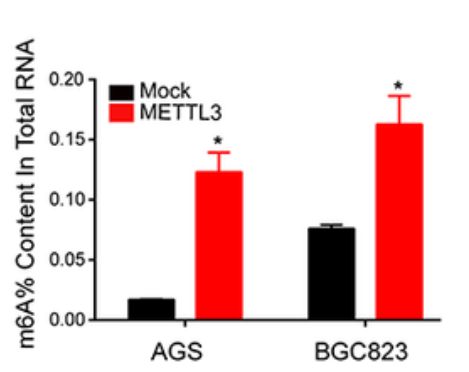

D

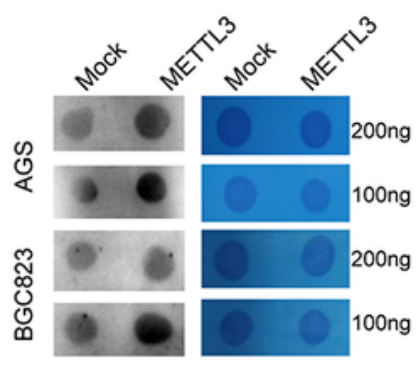

F
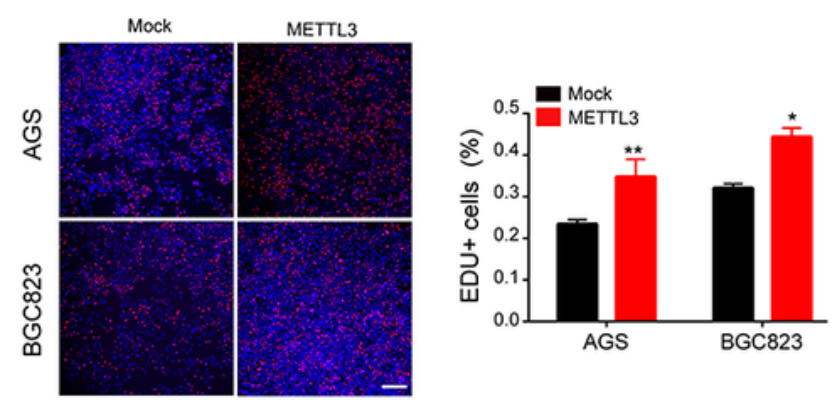

G

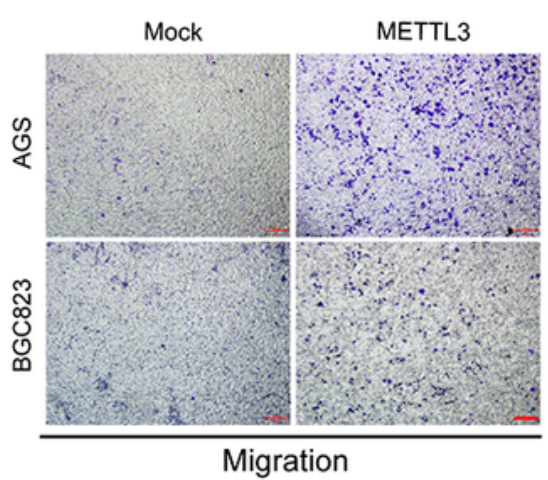

$\mathrm{H}$

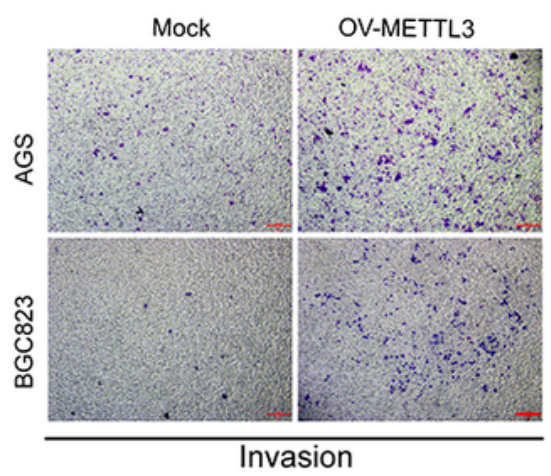

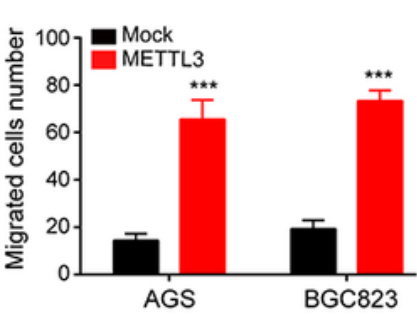

I
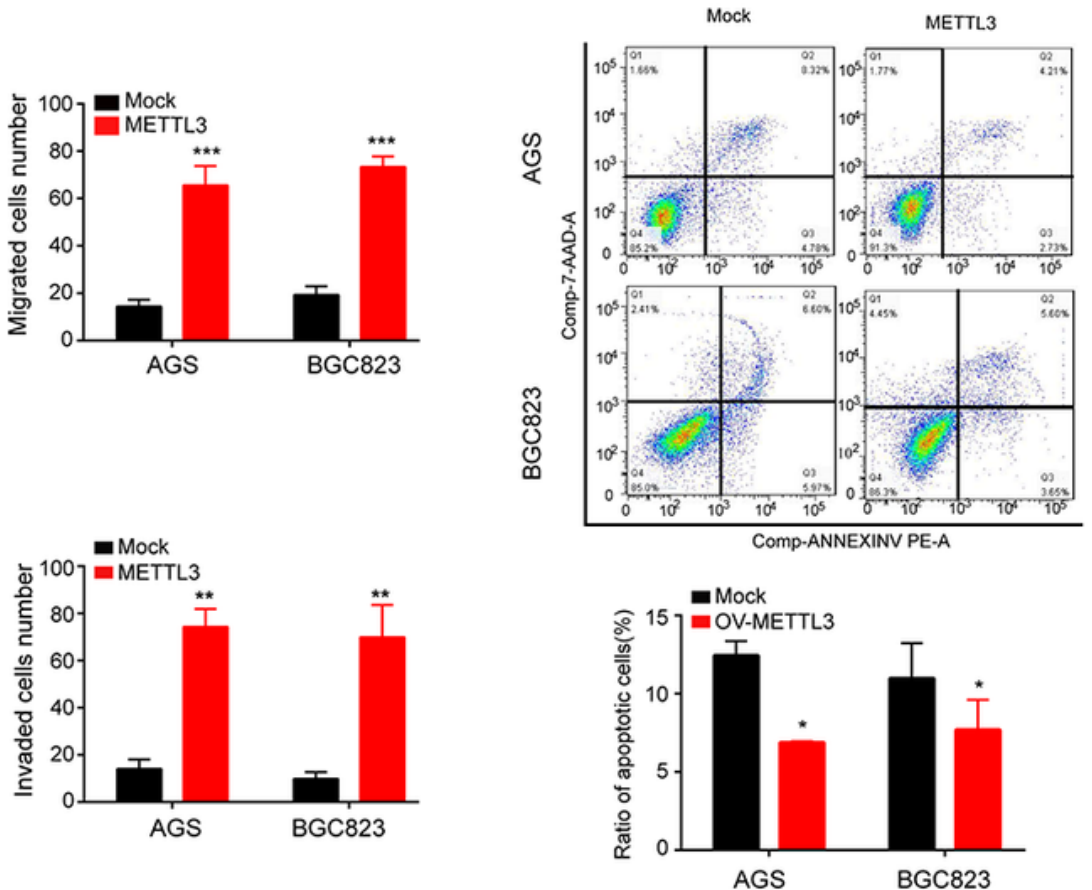

Figure 3

METTL3 promotes GC cell proliferation, migration and invasion, and inhibits apoptosis in vitro. $A$ and $B$ qRT-PCR and western blotting assay revealing the levels of METTL3 overexpression. C, RNA m6A levels were detected by colorimetric ELISA-like assay using the RNA m6A methylation quantification kit. D, RNAs isolated from mock or METTL3-overexpressing GC cells were used in dot blot analyses with an anti-m6A antibody. Methylene blue staining served as the loading control. E and F, CCK8 and EdU assays were used 
to analyze the proliferation of AGS and BGC823 cells after METTL3 overexpression. Scale bar, $200 \mu \mathrm{m}$. G and $\mathrm{H}$, Transwell assay illustrated that METTL3 overexpression facilitated the migration and invasion of AGS and BGC823 cells. Scale bars, 20 um. I, Annexin/PI staining followed by FACS analysis indicated that METTL3 overexpression inhibited apoptosis. ${ }^{*} p<0.05,{ }^{* *} p<0.01,{ }^{* *} p<0.001$

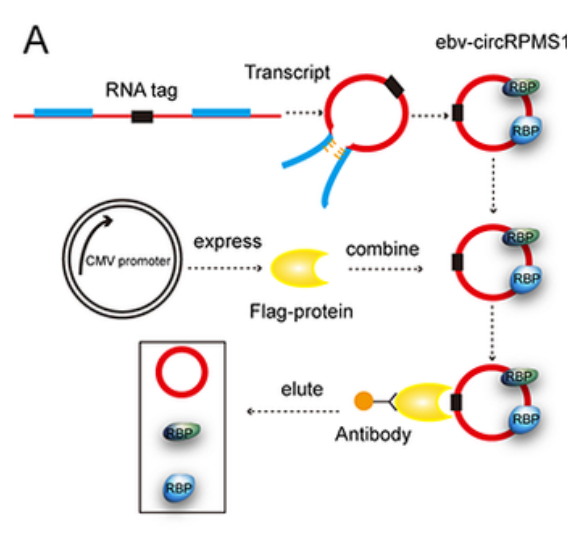

D

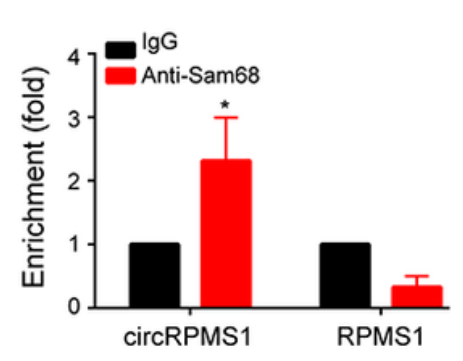

F

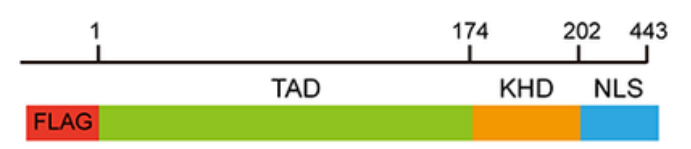

KD-WT FLAG

KD-1 FLAG

KD-2 FLAG
B

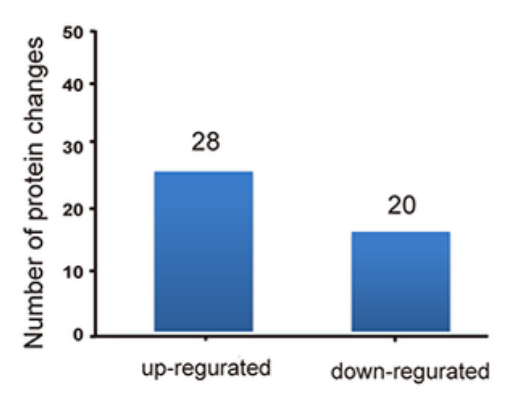

$\mathrm{E}$
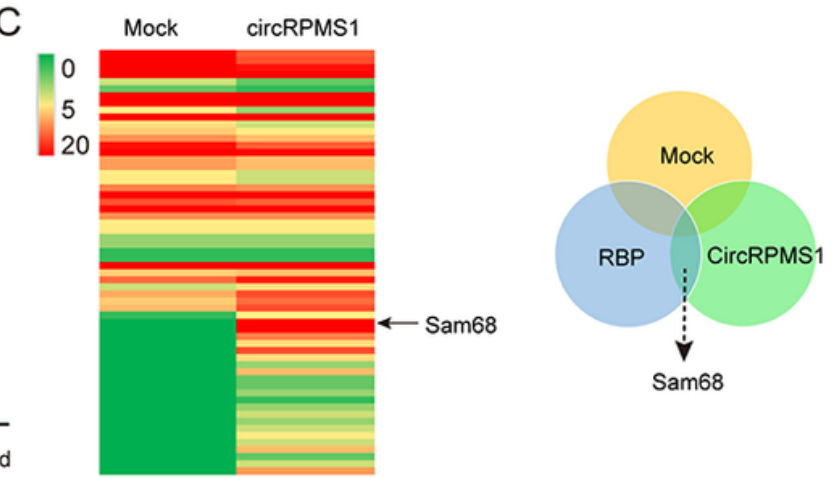

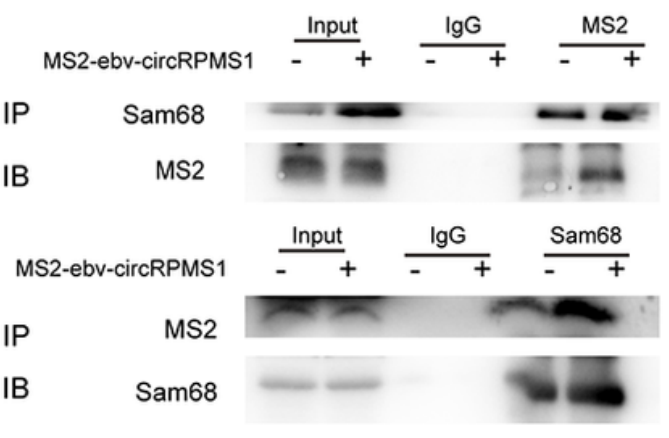

G

$\mathrm{H}$

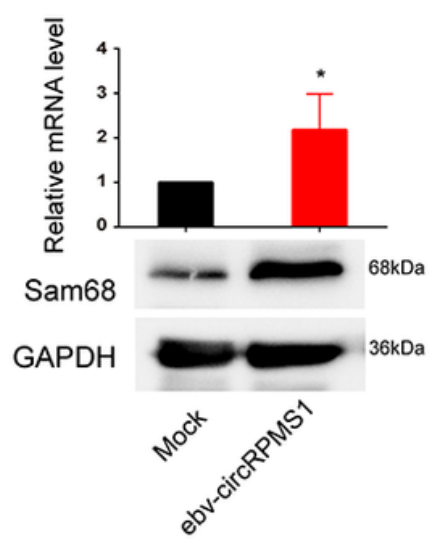

Figure 4

ebv-circRPMS1 directly interacts with Sam68 complex. A, Schematic diagram showing the process to generate RNA tag-labeled ebv-circRPMS1 by ligation of in vitro transcribed linear transcripts. B, RNA taglabeled ebv-circRPMS1 was incubated with SNU719 cell lysates, followed by mass spectrum identification. 48 proteins were pulled down by exogenous ebv-circRPMS1, including 28 up-regulated proteins and 20 down-regulated ones. C, Heat map showing the differentially expressed peptide. Venn diagram indicating the Sam68 was identified as a potential candidate for ebv-circRPMS1-interacting partner. D, RIP assay using anti-Sam68 showed that Sam68 precipitated ebv-circRPMS1 in SNU719 cell lysates. E, Pulldown assay confirmed that RNA tag-labeled ebv-circRPMS1 interacted with Flag-Sam68. F, 
Domain mapping assay indicated that the region of 174-202 aa in Sam68 was essential for the interaction with ebv-circRPMS1. G, RIP assay confirmed the interaction of Sam68 (174-202 aa) with ebvcircRPMS1. H, qRT-PCR and western blotting assay revealing the up-regulation of Sam68 after ebvcircRPMS1 overexpression.

A
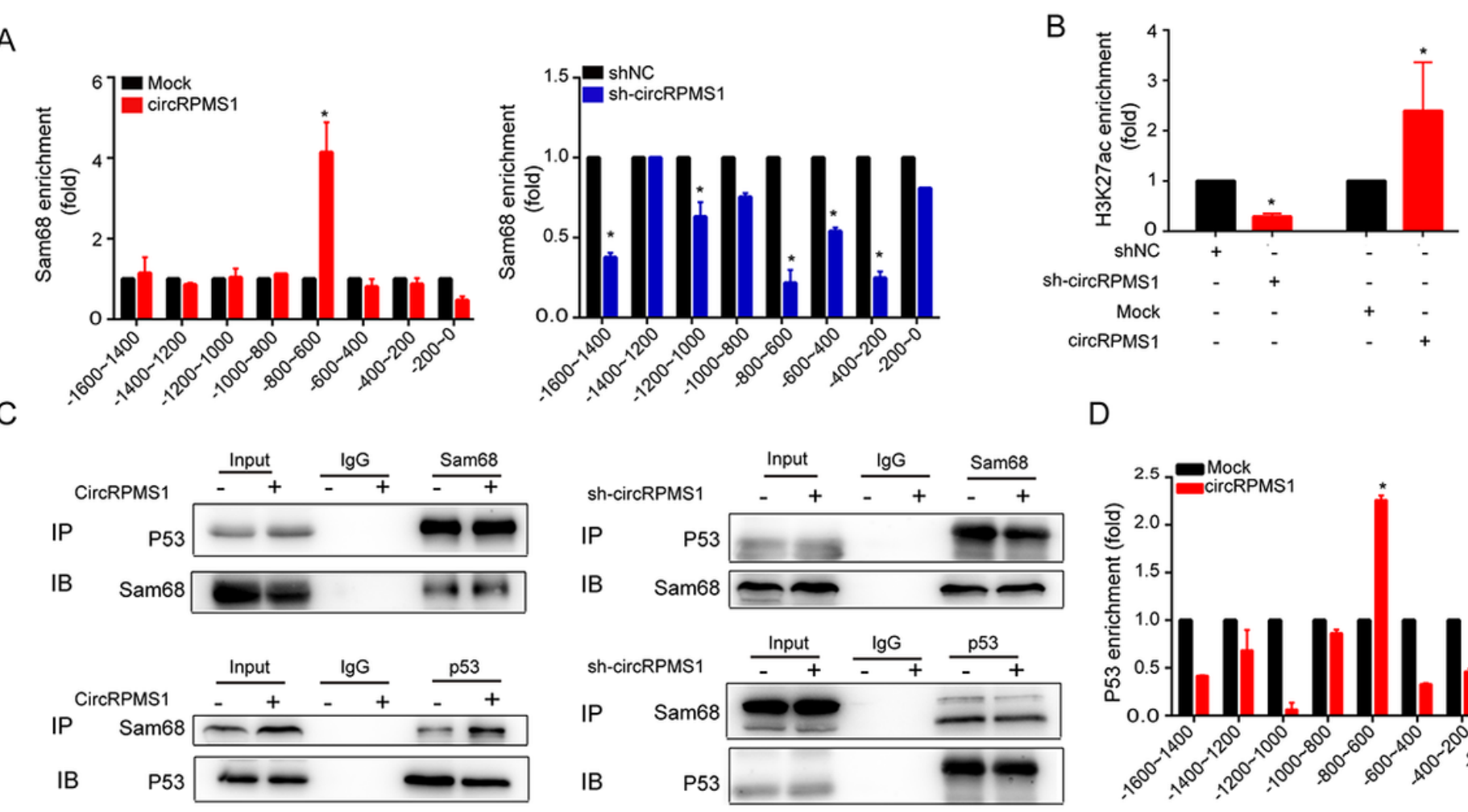

D

$\mathrm{E}$

$\mathrm{F}$

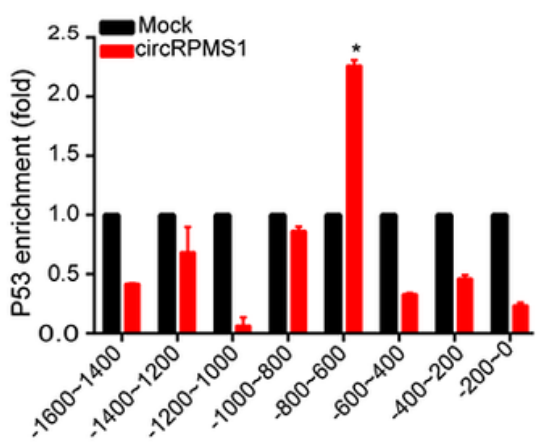

\section{E}

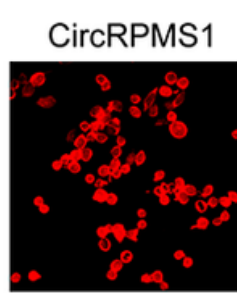

CircRPMS1
P53

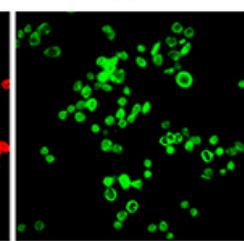

Sam68
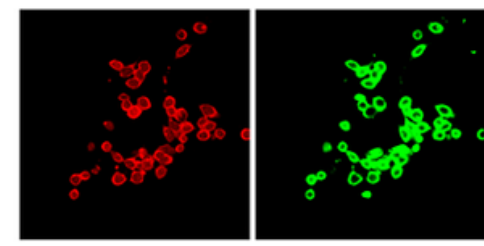

DAPI

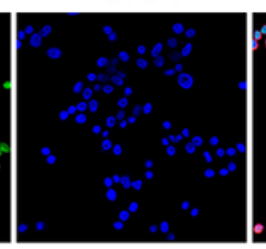

DAPI

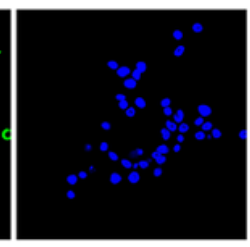

Merge

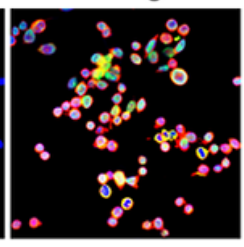

Merge

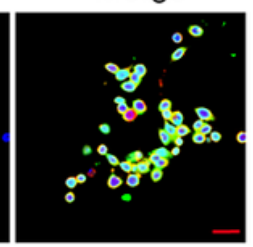

METTL3 promoter

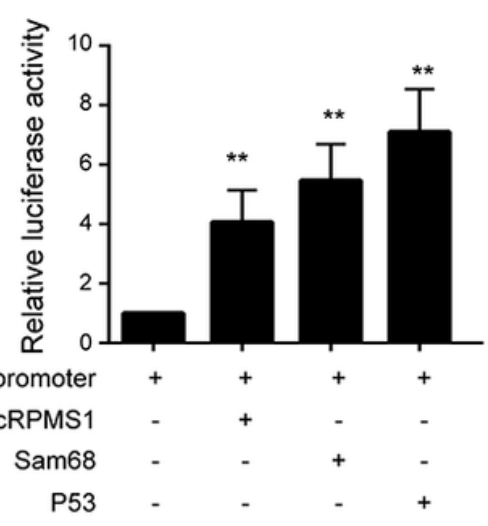

Figure 5

ebv-circRPMS1 recruits the Sam68 complex to activate METTL3 transcription. A, ChIP assay showed that Sam68 was enriched on METTL3 promoter, and ebv-circRPMS1 silencing attenuated the enrichment of Sam68 on METTL3 promoter. B, ChIP assay showed that depletion of ebv-circRPMS1 impaired the enrichment of active markers H3K27ac on METTL3 promoter. C, Co-IP assay showed that Sam68 interacts with P53. D, ChIP assay showed that P53 was enriched on METTL3 promoter. E, RNA-FISH 
assay verified the colocalization of ebv-circRPMS1, Sam68 and P53 in YCCEL1 cells. Scale bar, $20 \mu \mathrm{m}$. F, Luciferase reporter assay showed that overexpression of ebv-circRPMS1, Sam68, or P53 increased the luciferase activity. ${ }^{*} p<0.05,{ }^{* *} p<0.01$

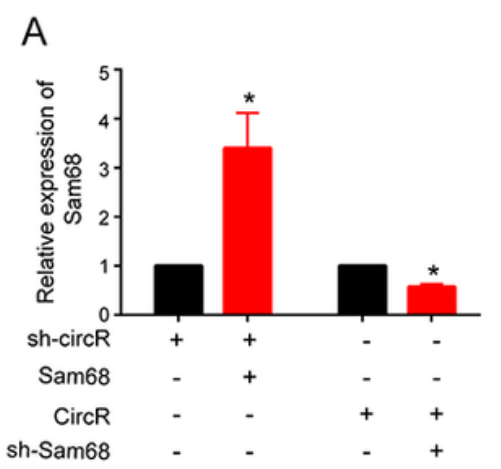

$E$

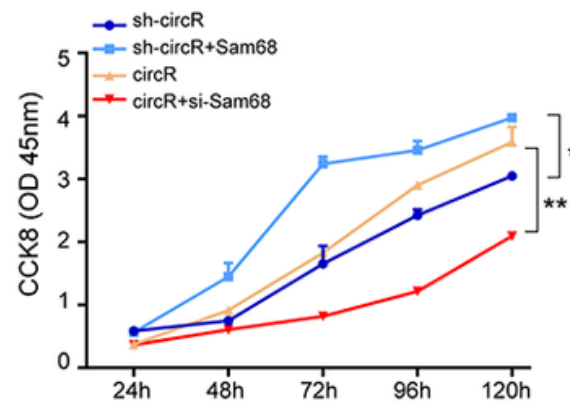

G

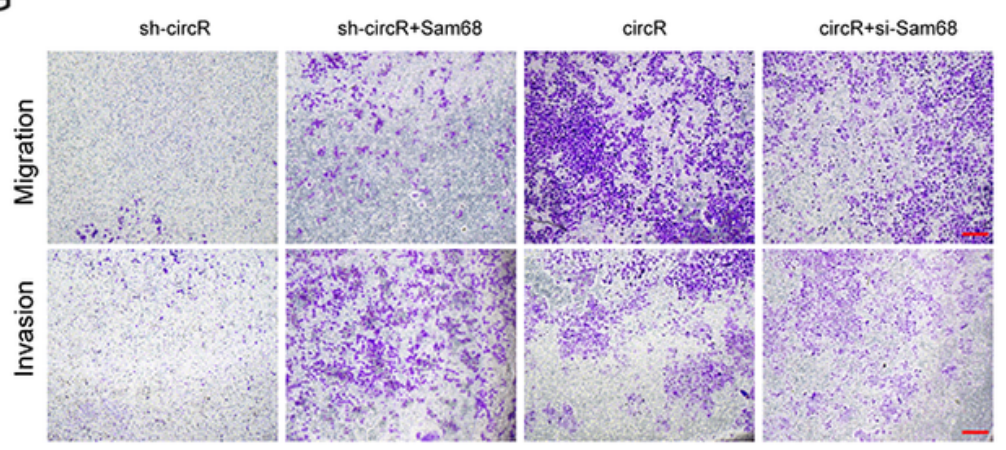

$\mathrm{H}$

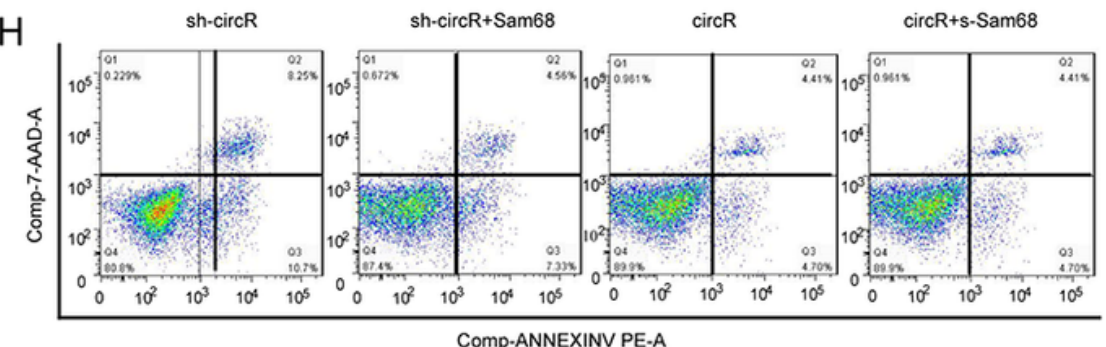

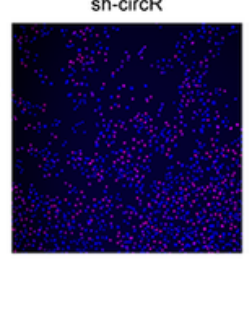

C

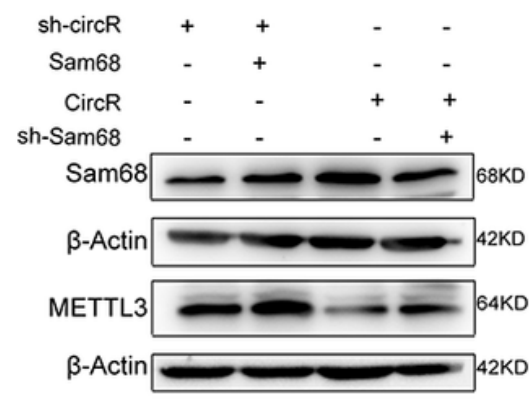

$\mathrm{F}$

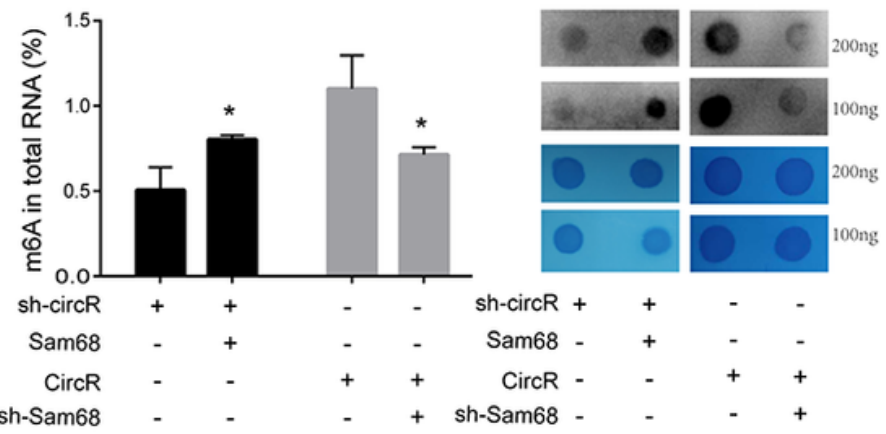

sh-circR+Sam68

circR

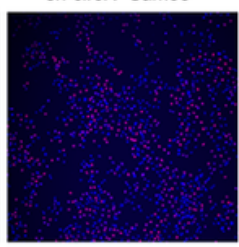

circR+si-Sam68
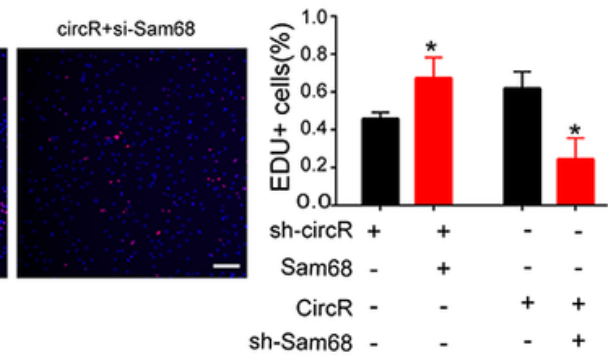
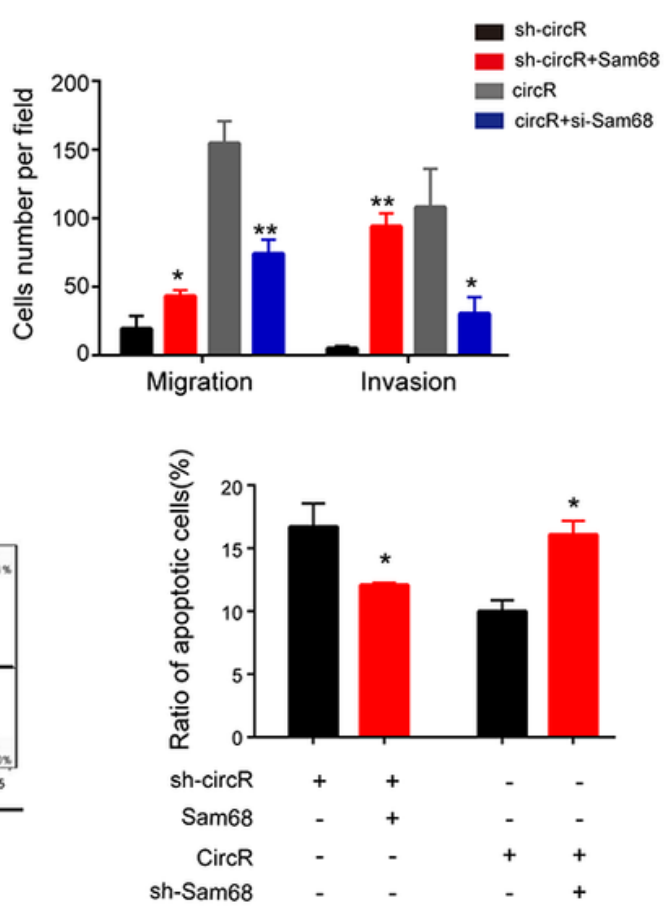

\section{Figure 6}

The ebv-circRPMS1/Sam68-METTL3 axis modulates GC progression. A and B, qRT-PCR and western blotting analysis of Sam68 expression in knockdown of ebv-circRPMS1 or overexpression ebv-circRPMS1 systems. C, RNA m6A levels were detected by colorimetric ELISA-like assay using the RNA m6A 
methylation quantification kit. D, RNAs isolated from GC cells were used in dot blot analyses with an antim6A antibody. Methylene blue staining served as the loading control. E and F, CCK8 and EdU assays were performed to detect cell proliferation. Scale bar, $200 \mu \mathrm{m}$. G and H, Restoration of Sam68 rescued the abilities of migration and invasion in ebv-circRPMS1 knockdown. Scale bars, $20 \mu \mathrm{m}$. I, Restoration of Sam68 reduced the apoptosis of YCCEL1 and AGS cells induced by ebv-circRPMS1 silencing. ${ }^{*} p<0.05$, $\star * p<0.01$

\section{A}
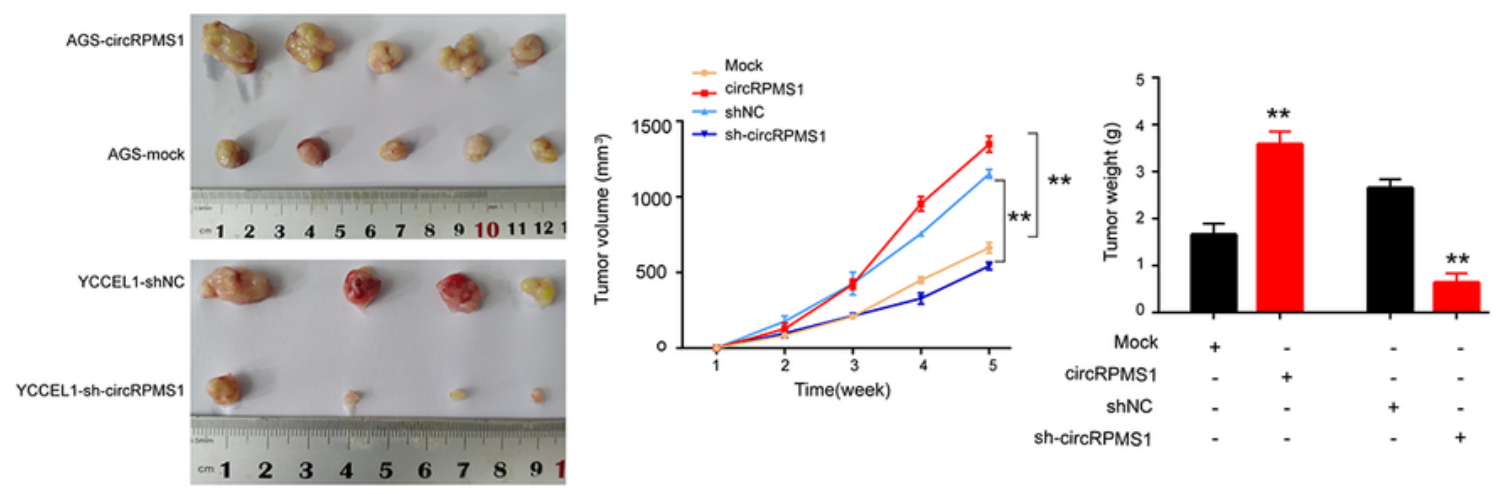

B

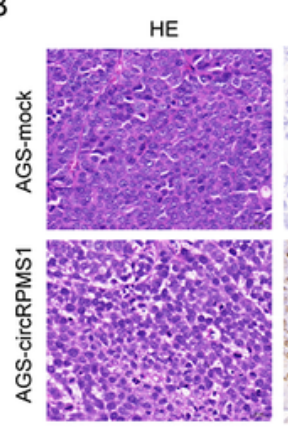

EBER
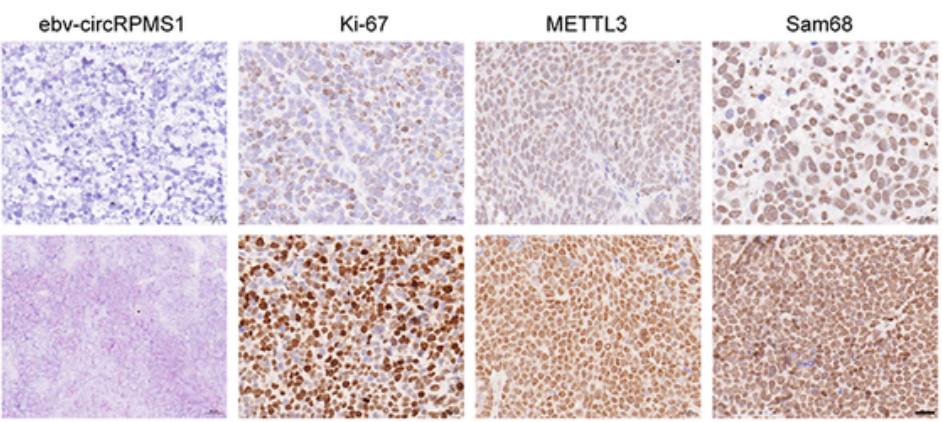

C

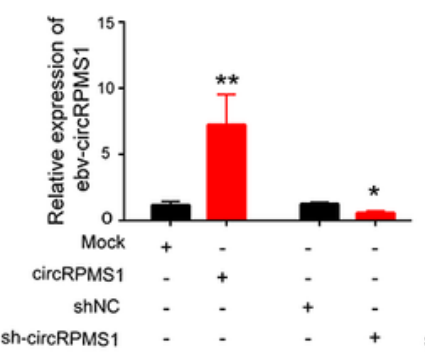

$\mathrm{E}$

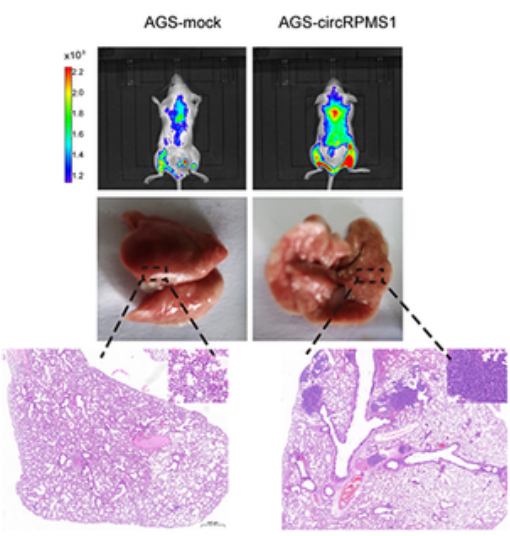

D

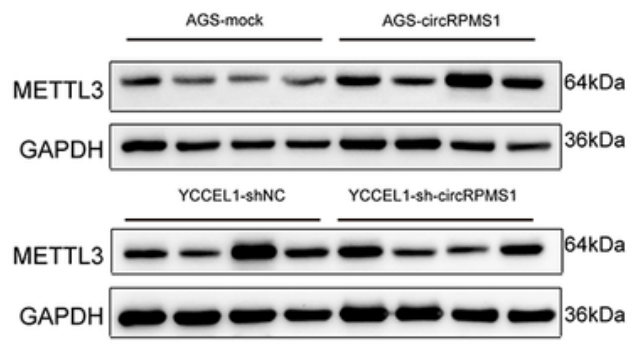


Effects of ebv-circRPMS1 on GC growth in vivo. A, Representative images of tumor tissues in each group were presented in the left. Tumor volumes were determined every 5 days. After sacrificed, the tumor weights were measured ( $n=5$ for each group). B, ISH analysis of EBER, RNAscope analysis of ebvcircRPMS1 and IHC analysis of METTL3, Ki67 and Sam68 expression in tumor tissues of each group. Scale bar, $20 \mu \mathrm{m}$. C, qRT-PCR analysis of ebv-circRPMS1 and METTL3 in tumor tissues. D, western blotting analysis of METTL3 in tumor tissues. E, left and middle, representative hematoxylin and eosin staining images; right, the number of metastatic nodules formed in the lungs of mice for each group $(n=$ 5 for each group). Scale bar, $500 \mu \mathrm{m} .{ }^{*} \mathrm{p}<0.05,{ }^{* \star} \mathrm{p}<0.01,{ }^{\star \star \star} \mathrm{p}<0.001$

A
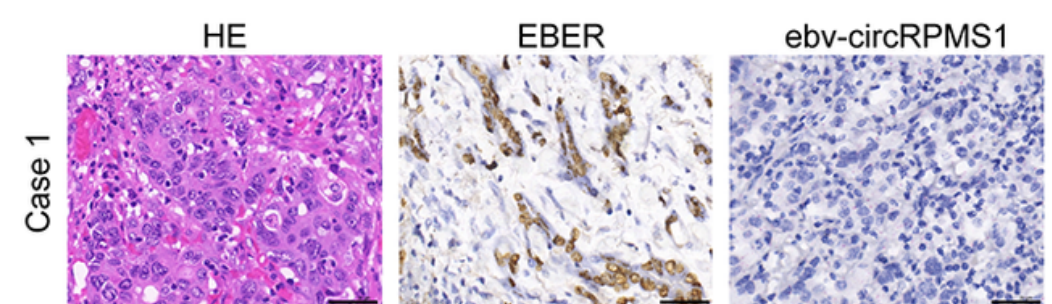

METTL3

Sam68
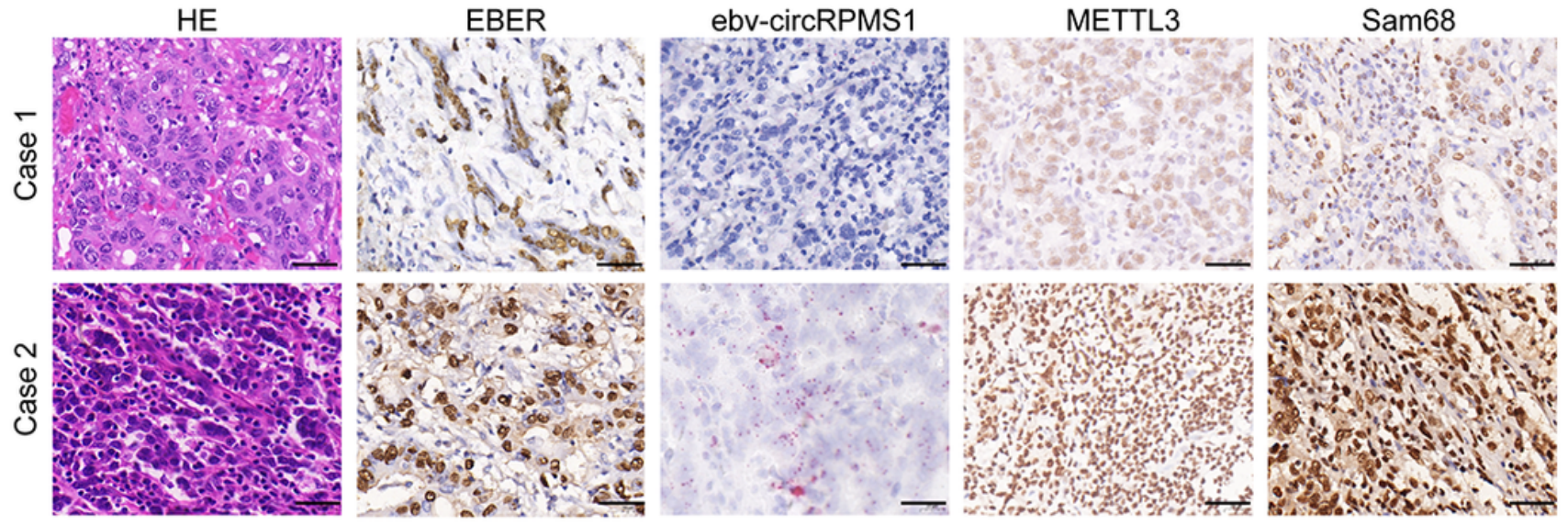

B
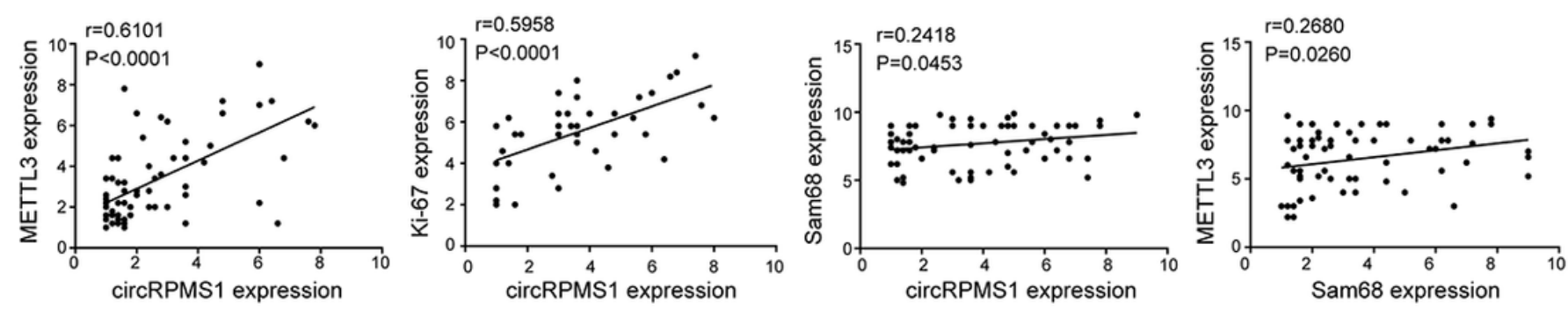

C
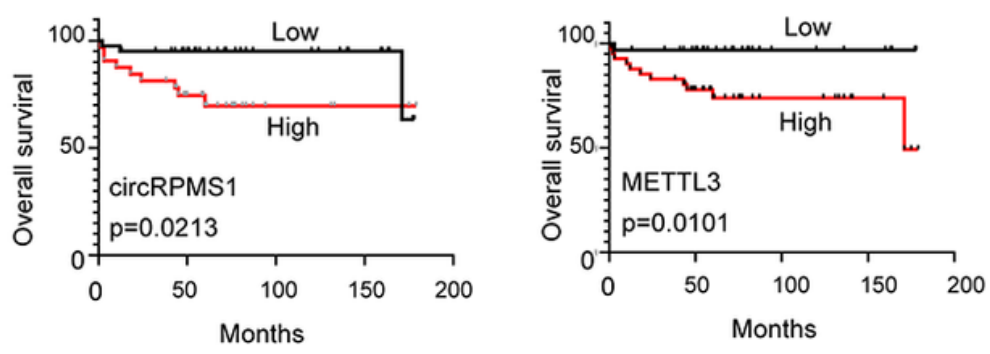

Figure 8

ebv-circRPMS1 is upregulated in EBVaGC tissues and positively correlated with poor prognosis. A, ISH analysis of EBER, RNAscope analysis of ebv-circRPMS1 and IHC analysis of METTL3 and Sam68 expression in EBVaGC tissues. Scale bars, $100 \mu \mathrm{m}$. B, ebv-circRPMS1 was positively correlated with 
METTL3 expression ( $r=0.6101, P \llbracket 0.0001), \mathrm{Ki}-67$ expression $(r=0.5958, \mathrm{P} \rrbracket 0.0001)$, and Sam68 expression $(r=0.2418, P=0.0453)$ in 70 EBVaGC tissues. Sam68 was positively correlated with METTL3 expression in 70 EBVaGC tissues $(r=0.2680, P=0.0260)$. C, Kaplan-Meier survival curves of OS based on ebv-circRPMS1 and METTL3 expression in 70 EBVaGC patients. All patients were divided into two groups based on the median level of ebv-circRPMS1 and METTL3, respectively. The log-rank test was used to calculate the significant level $(n=70)$.

A
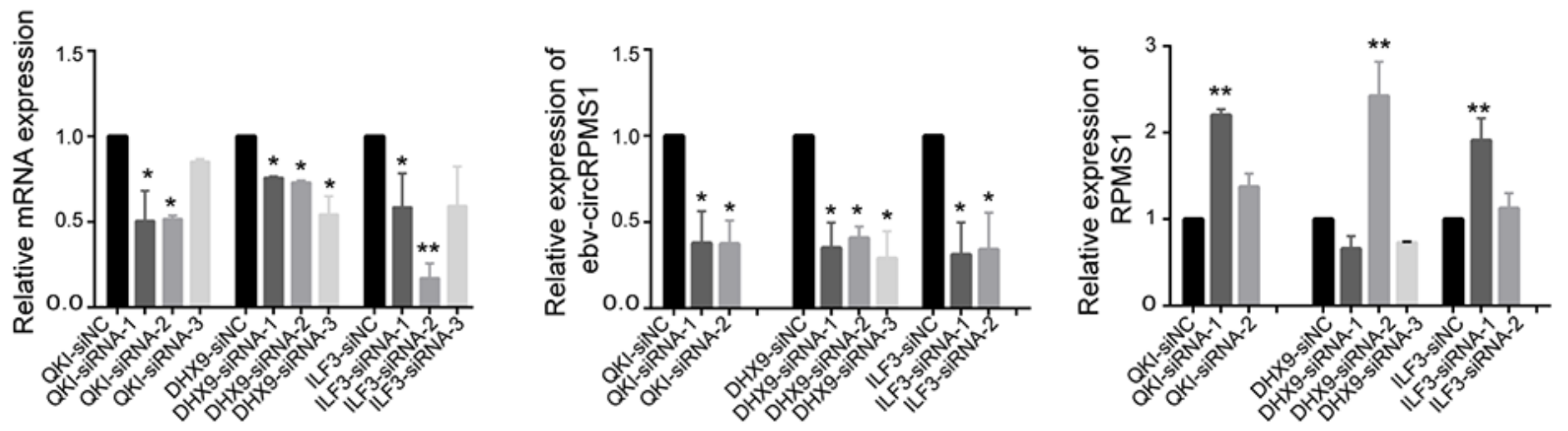

B

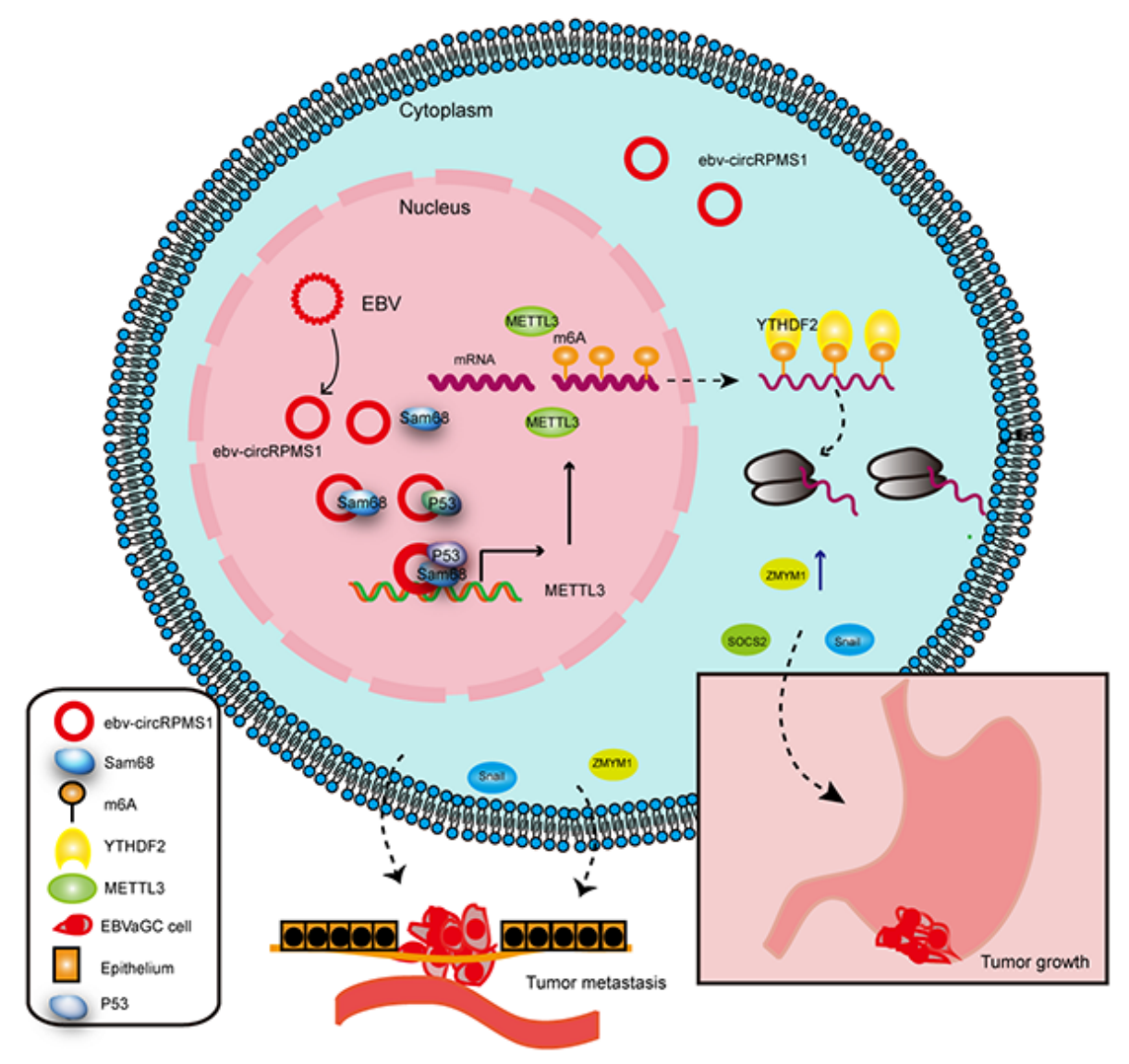

Figure 9

QKI, DHX9 and ILF3 are associated with ebv-circRPMS1 biogenesis. A, qRT-PCR assay revealing the levels of ebv-circRPMS1 and RPMS1 mRNA after QKI, DHX9 or ILF3 silencing. B, Schematic diagram showing 
the mechanisms underlying ebv-circRPMS1 promoted GC progression. ebv-circRPMS1 binds Sam68 to facilitate its interaction with p53, resulting in transactivation of METTL3 and transcriptional alteration of downstream target genes, which associated with metastasis and cancer progression. ${ }^{\star} p<0.05,{ }^{* \star} p<0.01$

\section{Supplementary Files}

This is a list of supplementary files associated with this preprint. Click to download.

- SupplementaryFigures.doc

- SupplementaryTables.doc 